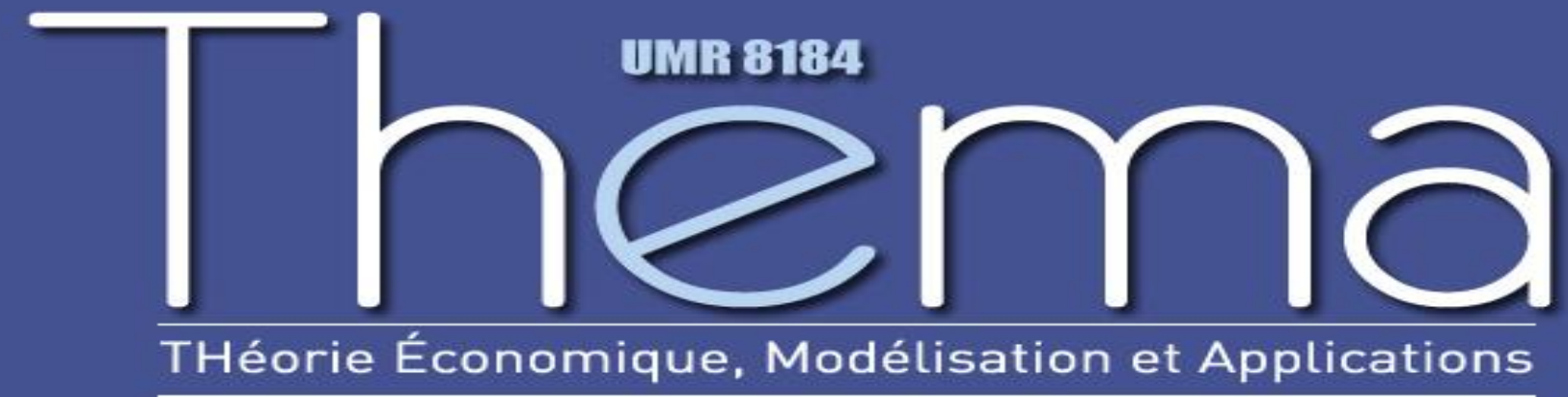

Thema Working Paper $\mathrm{n}^{\circ}$ 2012-28

Université de Cergy Pontoise, France

Noise-Independent Selection in Multidimensional Global Games

Marion Oury

March, 2012 


\title{
Noise-Independent Selection in Multidimensional Global Games *
}

\author{
Marion Oury ${ }^{\dagger}$
}

March 2012

\begin{abstract}
This paper examines many-player many-action global games with multidimensional state parameters. It establishes that the notion of noise-independent selection introduced by Frankel, Morris and Pauzner (Journal of Economic Theory 108 (2003) 1- 44) for onedimensional global games is robust when the setting is extended to the one proposed by Carlsson and Van Damme (Econometrica, 61, 989-1018). More precisely, our main result states that if an action profile of some complete information game is noise-independently selected in some one-dimensional global game, then it is also noise-independently selected in all multidimensional global games.
\end{abstract}

Keywords: equilibrium selection, global games, strategic complementarities, robustness. Journal of Economic Litterature: Classification Numbers: C72, D82.

*I am very grateful to Sylvain Chassang, Laurent Mathevet, Stephen Morris, Daisuke Oyama, Olivier Tercieux and Nicolas Vieille for helpful comments and suggestions. Of course, all errors or omissions are mine.

${ }^{\dagger}$ University of Cergy-Pontoise, THEMA, F-95000 Cergy-Pontoise, e-mail: marion.oury@u-cergy.fr, telephone number: +33134252257. 


\section{Introduction}

The global games framework, first proposed in Carlsson and Van Damme [2] (henceforth "CVD") for two-player two-action games, has been widely used to predict behaviour in games with strategic complementarities ${ }^{1}$. A global game extends a complete information game $g$ by a payoff function $u$ that depends on an additional state parameter $t$. In this approach, each agent receives a noisy private signal about the true state. Hence, at equilibrium, he's uncertain not only about his own payoff function but also -and more importantly- about other agents' beliefs and behavior. CVD's first result is that the set of rationalizable strategies shrinks to a unique equilibrium as the noise in private signals vanishes. They also established, as a second result, that the selected equilibrium is to play the risk-dominant strategy (Harsanyi and Selten [4]). Frankel, Morris and Pauzner [3] (henceforth "FMP") extended CVD's approach to manyplayer many-action games with strategic complementarities by showing that as the amplitude of the noise goes to zero, the limit uniqueness result of CVD holds: agents coordinate on some action profile that is a Nash equilibrium of the complete information game g. Nevertheless, in contrast with CVD, they restrict their analysis to settings where the state parameters belong to the real line and assume that actions are linearly ordered so that higher states lead to higher actions. Such an assumption is restrictive since agents involved in such complex coordination problems as speculative attacks often have to examine not only one but several criteria. In addition, the one-dimensional setting implies in particular that if the switch from one state to another gives to one agent an incentive to play a higher action, then it must also be the case for all the other agents. Yet, in most economic applications, it is not possible to exclude that different agents may focus on different criteria. In other terms, restricting to one-dimensional settings may imply too much homogeneity among the agents.

Regarding the second result of CVD, FMP provide a counter-example which shows that, unfortunately, in many-player many-action games, the selected action profile may depend on the fine details of the noise distribution. They thus define the notion of noise-independent selection: an action profile is said to be noise-independently selected at some state parameter if it is played at this state parameter regardless of the noise structure. Not being noiseindependent is a weakness for a given selection since the global games approach considers situations where the noise shrinks to zero : in general, there is no specific rationale for

\footnotetext{
${ }^{1}$ For a survey of the applied literature, see Morris and Shin [6] and Morris [5].
} 
choosing a noise structure rather than another. In a recent contribution, Basteck, Daniels and Heinemann [1] provide several positive results on the notion of noise-independent selection. First, they show that it is an intrinsic property of a complete information game : if a game of strategic complementarities is noise-independent under one (one-dimensional) global game embedding then it is also noise-independent under every other (one-dimensional) global game embedding. Second, they give simple sufficient conditions for noise-independent selection. In particular, they show that if an equilibrium is "robust to incomplete information", as defined by Kajii and Morris [5], then it will be noise-independently selected ${ }^{2}$. They also establish that the global game selection in a complete information game $g$ may be noise-independent if $g$ can be suitably decomposed into smaller noise-independent games. This result gives a simple tool to extend binary action environments to many-action games.

The present paper considers a setting generalizing both the framework of CVD and that of FMP: the complete information game $g$ has an arbitrary number of players and actions and the state parameter $t$ is multidimensional. In addition, in contrast with FMP, it is not assumed that for each state parameter $t$ in the support of the prior distribution, the complete information game associated with $t$ is supermodular. Our main result shows the coherence and robustness of the notion of noise-independent selection: if the action profile $a^{\star}$ is noiseindependently selected in one-dimensional global games, then it is also noise-independently selected in all multidimensional global games.

\section{$2 \quad$ Setting and Definitions}

In this paper, we consider games with a finite set of players $I$, who have finite and linearly ordered action sets $A_{i \in I}$. We write $C$ for the cardinal of the set $\cup_{i \in I} A_{i}$. We define the joint action space $A$ as $\prod_{i \in I} A_{i}$ and write $A_{-i}$ for $\prod_{j \neq i} A_{j}$. We say that $a=\left(a_{i}\right)_{i \in I} \in A$ is weakly greater than $a^{\prime}=\left(a_{i}^{\prime}\right)_{i \in I} \in A$ if $a_{i} \geq a_{i}^{\prime}$ for all $i \in I$ and write $a \geq a^{\prime}$.

\footnotetext{
${ }^{2}$ In $2 \times 2$ games, the risk-dominant equilibrium is robust. Characterizations for more general games have been given by Morris and Ui [8] and Oyama and Tercieux [9]. A heuristic argument establishing that robustness implies noise-independence may be found in Morris and Shin [7]. Oyama and Takahashi [10] showed recently that noise-independent selection (for one-dimensional global games) does not imply robustness.
} 


\subsection{N-dimensional Global Games}

Fix $N \geq 1$. For all $x \in \mathbb{R}^{N}$, we write $\|x\|$ for the euclidean norm of $x$ and for each $\mu>0$, we write $B^{\mu}(x)$ for the closed ball of radius $\mu$ around $x$. A global game $G_{\nu}(u, \phi, \psi)$ is an incomplete information game where payoffs depend on a random variable $t \in \mathbb{R}^{N}$, called the state parameter, which is distributed according to a continuous density $\psi$, called the prior distribution. The individual payoffs are given by $u_{i}\left(a_{i}, a_{-i}, t\right)$, where $a_{i}$ denotes $i$ 's action and $a_{-i}$ denotes the opposing action profile. They are assumed to be continuous in the state parameter $t . \phi$ is a tuple of atomless densities that we refer to as the noise structure. Each player $i \in I$ observes a $N$-dimensional private signal $x_{i}=t+\nu \eta_{i}$ about $t$, where $\nu>$ is a scale factor and $\eta_{i}$ an error that is distributed according to the density $\phi_{i}$ whose support is a subset of $B^{\frac{1}{2}}(0)$. The random variables $\left\{t, \eta_{1}, \ldots, \eta_{I}\right\}$ are independently distributed.

A strategy for player $i$ is a measurable function $s_{i}: \mathbb{R}^{N} \rightarrow A_{i}$ and a strategy profile $s$ is a tuple of such strategies, $s=\left(s_{i}\right)_{i \in I}$. A strategy profile $s$ is increasing if each component $s_{i}$ is increasing and left (right)-continuous if each $s_{i}$ is left (right)-continuous. If $x$ is a tuple of signals $\left(x_{i}\right)_{i \in I}$, then $s(x)$ denotes the action profile $\left(s_{i}\left(x_{i}\right)\right)_{i \in I}$. Slightly abusing notation, for $x \in \mathbb{R}$, we also denote the action profile given by $\left(s_{i}(x)\right)_{i \in I}$ by $s(x)$. We say that the strategy profile $s$ is weakly greater than the strategy profile $s^{\prime}$ if $s(x) \geq s^{\prime}(x)$ for all $x \in \mathbb{R}$ and write $s \geq s^{\prime}$.

As in FMP, our main solution concept is iterative strict dominance. First we eliminate pure strategies that are strictly dominated, as rational players will never pick (i.e., put positive weight on) such strategies. Then we eliminate a player's pure strategies that are strictly dominated if her opponents are known to mix only over the pure strategies that survived the prior round of elimination; and so on.

\subsection{Embedding a Complete Information Game in a Global Game}

Let a complete information game $g$ be specified by its payoff function $g_{i \in I}\left(a_{i}, a_{-i}\right)$ with $g_{i}$ : $A \rightarrow \mathbb{R}$ for each player $i$. For each $i \in I, a_{i}, a_{i}^{\prime} \in A_{i}$ and $a_{-i} \in A_{-i}$, we note $\Delta g_{i}\left(a_{i} \rightarrow a_{i}^{\prime}, a_{-i}\right)$ for the difference in payoff of player $i$ from playing $a_{i}^{\prime}$ versus $a_{i}$ when the action profile of his opponents is $a_{-i}$, that is,

$$
\Delta g_{i}\left(a_{i} \rightarrow a_{i}^{\prime}, a_{-i}\right)=g_{i}\left(a_{i}^{\prime}, a_{-i}\right)-g_{i}\left(a_{i}, a_{-i}\right)
$$


A game $g$ is a game of strategic complementarities if greater opposing action profiles make greater action more appealing, or more precisely, if for all $i, a_{i} \geq a_{i}^{\prime}$ and $a_{-i} \geq a_{-i}^{\prime}$,

$$
\Delta g_{i}\left(a_{i} \rightarrow a_{i}^{\prime}, a_{-i}^{\prime}\right) \geq \Delta g_{i}\left(a_{i} \rightarrow a_{i}^{\prime}, a_{-i}\right) .
$$

We say that the global game $G_{\nu}(u, \phi, \psi)$ embeds the complete information game $g$ at state parameter $t^{\star}$ if there exist $\tilde{t}^{\star} \in[0,1]$ and a continuous function $\theta:[0,1] \rightarrow \mathbb{R}^{N}$ such that (i) $\theta\left(\tilde{t}^{\star}\right)=t^{\star}$ and (ii) the four assumptions below are satisfied.

By a slight abuse of notation, for each $\mu>0$, we write $B^{\mu}(\Theta)$ for the set of $y \in \mathbb{R}^{N}$ such that there exists $\tilde{t} \in[0,1]$ with: $\|y-\theta(\tilde{t})\| \leq \mu$.

Assumption 1. There exists $\bar{\mu}>0$ such that for all state parameters $t \in B^{\bar{\mu}}(\Theta), \psi(t)>0$.

Assumption 2. For all $t \in B^{\bar{\mu}}(\Theta)$, the complete information game associated with the payoff function $u(\cdot, t)$ is a game of strategic complementarities.

For each player $i$, write $u_{i}^{\theta}: A \times[0,1] \rightarrow \mathbb{R}$ for the function defined by $u_{i}^{\theta}(a, \tilde{t})=u_{i}(a, \theta(\tilde{t}))$, for all $a \in A$ and $\tilde{t} \in[0,1]$.

Assumption 3. The payoff function $u^{\theta}$ satisfies strict monotonicity. That is, there is a $K>0$ such that for all $i, a_{-i}, a_{i}<a_{i}^{\prime}$ and $\tilde{t}, \tilde{t}^{\prime} \in[0,1]$ with $\tilde{t}^{\prime}>\tilde{t}$,

$$
\Delta u_{i}^{\theta}\left(a_{i} \rightarrow a_{i}^{\prime}, a_{-i}, \tilde{t}^{\prime}\right)-\Delta u_{i}^{\theta}\left(a_{i} \rightarrow a_{i}^{\prime}, a_{-i}, \tilde{t}\right) \geq K\left(\tilde{t}^{\prime}-\tilde{t}\right) .
$$

Assumption 4. Extreme values of $\tilde{t}$ make the extreme actions dominant choices. More precisely, there exists $L$ such that if $\tilde{t}<L$ then, for each player $i$, each action $a_{i} \backslash \min A_{i}$ and each action profile $a_{-i} \in A_{-i}$,

$$
\Delta u_{i}^{\theta}\left(a_{i} \rightarrow \min A_{i}, a_{-i}, \tilde{t}\right)>0
$$

Symmetrically, if $\tilde{t}>1-L$ then, for each player $i$, each action $a_{i} \backslash \max A_{i}$ and each action profile $a_{-i} \in A_{-i}$,

$$
\Delta u_{i}^{\theta}\left(a_{i} \rightarrow \max A_{i}, a_{-i}, \tilde{t}\right)>0 .
$$

\subsection{Noise-Independent Selection in One-dimensional Global Games}

In this subsection, we assume that $N=1$. In this specific case, it is easily checked that the framework presented in Subsection 2.2 is equivalent to the one proposed by FMP and their main result applies: 
Theorem 1 (FMP, Theorem 1). The global game $G_{\nu}(u, \phi, \psi)$ has an essentially unique strategy profile surviving iterative strict dominance in the limit as $\nu \rightarrow 0$. More precisely, there exists an increasing pure strategy profile $s^{\star}$ such that if, for each $\nu>0, s_{\nu}$ is a pure strategy profile that survives iterative strict dominance in $G_{\nu}(u, \phi, \psi)$, then for each player $i$, $\lim _{\nu \rightarrow 0} s_{\nu, i}\left(x_{i}\right)=s_{i}^{\star}\left(x_{i}\right)$ for all $x_{i}$, except possibly at the finitely many discontinuities of $s_{i}^{\star}$.

We also recall the definition that FMP introduced for noise-independent selection.

Definition 1. We say that the action profile $a^{\star}$ is noise-independently selected at some state parameter $t^{\star}$ if $s^{\star}\left(t^{\star}\right)=a^{\star}$, regardless of the noise structure $\phi$.

FMP established that $s^{\star}$ does not depend on the prior distribution $\psi$. In addition, Basteck, Daniels and Heinemann [1] showed that the global game selection is independent of the payoff function $u$ of the global game embedding. Consequently, noise-independence (for onedimensional global games) is an intrinsic property of a complete information game.

\section{Main Result}

When $N>1$, the argument of FMP for limit uniqueness does not hold and we cannot use the strategy profile $s^{\star}$ for the definition of noise-independence. Hence, we adopt instead the following equivalent formulation.

Definition 2. We say that the action profile $a^{\star}$ is noise-independently selected in $N$-dimensional global games embedding the complete information game $g$ if for each $N$-dimensional global game $G_{\nu}(u, \phi, \psi)$ embedding $g$ at a state parameter $t^{\star}$, there exists $\bar{\nu}>0$ such that for each $\nu<\bar{\nu}$ and each strategy profile $s_{\nu}$ surviving iterative strict dominance in $G_{\nu}(u, \phi, \psi)$, $s_{\nu}\left(t^{\star}\right)=a^{\star}$.

We now state our main result.

Theorem 2. If the action profile $a^{\star}$ is noise-independently selected in 1-dimensional global games embedding the complete information game $g$, then $a^{\star}$ is also noise-independently selected in $N$-dimensional global games embedding $g$ for all $N>1$. 


\section{Proof}

Fix $N>1$, a $N$-dimensional global game $G_{\nu}(u, \phi, \psi)$ and a parameter function $\theta:[0,1] \rightarrow$ $\mathbb{R}^{N}$. We note $E_{\nu}\left(\Delta u_{i}\left(a_{i}^{\prime} \rightarrow a_{i}\right) \mid x_{i}, s_{-i}\right)$ for the expected difference in utility of player $i$ from playing $a_{i}$ versus $a_{i}^{\prime}$ against the strategy profile $s_{-i}$ when he receives the signal $x_{i}$ in $G_{\nu}(u, \phi, \psi)$. We will also use the notation $\mathbb{P}_{\nu}\left(F \mid x_{i}\right)$ (resp. $\left.\mathbb{P}_{\nu}(F \mid t)\right)$ to refer to the probability of an event $F$ conditional on the signal $x_{i}$ (resp. the state parameter $t$ ).

Let us write $V$ for the set of unit vectors $v \in \mathbb{R}^{N}$. For any $v \in V$ and $d \in \mathbb{R}$, we set:

$$
L(v, d)=\left\{y \in \mathbb{R}^{N} \mid y \cdot v=d\right\},
$$

where the symbol $\cdot$ denotes the dot product in $\mathbb{R}^{N}$. Moreover, for each player $i$, we let the density function $\phi_{i}^{v}:\left[-\frac{1}{2}, \frac{1}{2}\right] \rightarrow \mathbb{R}^{+}$be such that for all $d \in\left[-\frac{1}{2}, \frac{1}{2}\right], \phi_{i}^{v}(d)$ is the density of probability associated with the event $\eta_{i} \in L(v, d)$. We note $\Phi_{i}^{v}$ for the distribution function associated with $\phi_{i}^{v}$.

For each unit vector $v \in \mathbb{R}^{N}$, we write $G^{\theta}(\nu, v)$ for the one-dimensional global game associated with the noise structure $\left(\phi_{i}^{v}\right)_{i \in I}$, the payoff function $\left(u_{i}^{\theta}\right)_{i \in I}$, the scale factor $\nu$ and where the payoff parameter $t$ is drawn uniformly from the interval $[0,1]$. In addition, applying FMP's main result, we note $s_{\theta}^{\star}(v)$ for the essentially unique strategy profile surviving iterative strict dominance in $G^{\theta}(\nu, v)$ as the noise shrinks to zero. Notice that if action profile $a^{\star}$ is noise-independently selected in one dimensional global games at state parameter $\tilde{t}^{\star}$, then for each unit vector $v, s_{\theta}^{\star}(v)$ takes the value $a^{\star}$ at $\tilde{t}^{\star}$. Consequently, Theorem 2 directly follows from Theorem 3 below.

Theorem 3. Let $\tilde{t}^{\star} \in[0,1]$ and $a^{\star} \in A$ be such that for all unit vectors $v, s_{\theta}^{\star}(v)$ takes the value $a^{\star}$ at $\tilde{t}^{\star}$. Then, there exists $\nu^{\star}>0$ such that for each $\nu<\nu^{\star}$, each pure strategy profile $s_{\nu}$ surviving iterative strict dominance in $G_{\nu}(u, \phi, \psi)$ must satisfy $s_{\nu}\left(\theta\left(\tilde{t}^{\star}\right)\right)=a^{\star}$.

Proof. We will establish that there exists $\nu^{\star}>0$ such that for each $\nu<\nu^{\star}$, each strategy profile $s_{\nu}$ surviving iterative strict dominance in $G_{\nu}(u, \phi, \psi)$ must satisfy $s_{\nu}\left(\theta\left(\tilde{t}^{\star}\right)\right) \leq a^{\star}$. (A symmetric argument yields that $s_{\nu}\left(\theta\left(\tilde{t}^{\star}\right)\right) \geq a^{\star}$.) As in FMP, we introduce the notion of a simplified global game $G_{\star}^{\theta}(\nu, v)$ that differs from $G^{\theta}(\nu, v)$ in that each player $i$ 's payoff depends directly on her signal $x_{i}$ rather than on the state $\tilde{t}$. Following notations similar to those used for the multidimensional global game $G_{\nu}(u, \phi, \psi)$, we let $E_{\nu}^{v}\left(\Delta u_{i}^{\theta}\left(a_{i}^{\prime} \rightarrow a_{i}\right) \mid x_{i}, s_{-i}\right)$ denote the expected difference in utility of player $i$ from playing $a_{i}$ versus $a_{i}^{\prime}$ against the 
strategy profile $s_{-i}$ when he receives the signal $x_{i}$ in $G_{\star}^{\theta}(\nu, v)$. We also write $\mathbb{P}_{\nu}^{v}\left(E \mid x_{i}\right)$ (resp. $\mathbb{P}_{\nu}^{v}(E \mid \tilde{t})$ ) for the probability of an event $E$ conditional on the signal $x_{i}$ (resp. the state parameter $\tilde{t}$ ) in $G_{\star}^{\theta}(\nu, v)$. For each $\nu>0$, we know (FMP, Lemma 4) that there exists an essentially unique strategy profile surviving iterative strict dominance in $G_{\star}^{\theta}(\nu, v)$ and we write $s^{\theta}(\nu, v)=\left\{s_{i}^{\theta}(\nu, v)\right\}_{i \in I}$ for its right-continuous version. For each $i$, we define: $A_{i}^{+}=\left\{a_{i} \in A_{i} \mid a_{i} \geq a_{i}^{\star}\right\}$ and $A_{i}^{-}=\left\{a_{i} \in A_{i} \mid a_{i}<a_{i}^{\star}\right\}$. In addition, for each increasing strategy profile $s$ in a one-dimensional global game, we set:

$$
x\left(a_{i}, s\right)=\sup \left\{x \in[0,1] \mid s_{i}(x) \leq a_{i}\right\},
$$

for each $a_{i} \in \cup_{i \in I} A_{i}$ and $x\left(a_{-i}, s\right)=\left(x\left(a_{j}, s\right)\right)_{j \neq i}$. The fact that the simplified global game $G_{\star}^{\theta}(\nu, v)$ converges toward $G^{\theta}(\nu, v)$ as the noise vanishes allows to establish Proposition 1 below. (The proof is standard but provided in Appendix for the sake of completeness.)

Proposition 1. There exist $\bar{\nu}^{\star}$ and $\lambda^{\star}>0$ such that for each $i \in I, \bar{\nu}<\bar{\nu}^{\star}$ and unit vector $v \in \mathbb{R}^{N}:$

- $x\left(a_{i}, s^{\theta}(\bar{\nu}, v)\right)>\tilde{t}^{\star}+\lambda^{\star}$, for all $a_{i} \in A_{i}^{+}$and,

- $x\left(a_{i}, s^{\theta}(\bar{\nu}, v)\right)<\tilde{t}^{\star}-\lambda^{\star}$, for all $a_{i} \in A_{i}^{-}$.

Proof. See Appendix.

We now pick some $\bar{\nu}<\min \left(\bar{\nu}^{\star}, \lambda^{\star}, L\right)$. (In the sequel of the proof, the scale factor $\bar{\nu}$ will sometimes be omitted from notations). We first build a "compressed" version of $s^{\theta}(\bar{\nu}, v)$ for each unit vector $v$.

Lemma 1. For each $\nu<\bar{\nu}$ and each $v \in V$, there exists a strategy profile $s_{c}^{\theta}(\bar{\nu}, \nu, v)$ in $G_{\star}^{\theta}(\nu, v)$ such that for each $a_{i} \in \cup_{i \in I} A_{i}^{+}$,

1. $\tilde{t}^{\star} \leq x\left(a_{i}, s_{c}^{\theta}(\bar{\nu}, \nu, v)\right)<\tilde{t}^{\star}+C \nu$, (where we recall that $C$ denotes the cardinal of the set $\left.\cup_{i \in I} A_{i}\right)$ and,

2. $\mathbb{P}_{\nu}^{v}\left(x_{-i} \leq x\left(a_{-i}, s_{c}^{\theta}(\bar{\nu}, \nu, v)\right) \mid x\left(a_{i}, s_{c}^{\theta}(\bar{\nu}, \nu, v)\right)\right)=\mathbb{P}_{\bar{\nu}}^{v}\left(x_{-i} \leq x\left(a_{-i}, s^{\theta}(\bar{\nu}, v)\right) \mid x\left(a_{i}, s^{\theta}(\bar{\nu}, v)\right)\right)$, for all $a_{-i} \in A_{-i}$.

Proof. Since $s^{\theta}(\bar{\nu}, v)$ is increasing and the joint action space $A$ is finite, we can identify $s^{\theta}(\bar{\nu}, v)$ with a finite sequence $z_{1}(v), z_{2}(v), \ldots, z_{k}(v)$ with $k \leq C$ of jump points, at which players switch to greater action profiles. Now, for each $\nu>0$, define recursively the sequence 
$z_{1}^{\prime}(\nu, v), \ldots, z_{k}^{\prime}(\nu, v)$ as follows. Set $z_{1}^{\prime}(\nu, v)=\tilde{t}^{\star}$ and, for each $n>1, z_{n}^{\prime}(\nu, v)=z_{n-1}^{\prime}(\nu, v)+\nu$ if $z_{n}(v)-z_{n-1}(v) \geq \bar{\nu}$ and $z_{n}^{\prime}(\nu, v)=z_{n-1}^{\prime}(\nu, v)+\frac{\nu}{\bar{\nu}}\left(z_{n}(v)-z_{n-1}(v)\right)$ otherwise. Finally, let $s_{\theta}^{c}(\bar{\nu}, \nu, v)$ be the right-continuous increasing strategy profile such that:

- For each $a_{i} \in \cup_{i \in I} A_{i}^{-}, x\left(a_{i}, s_{c}^{\theta}(\bar{\nu}, \nu, v)\right)=x\left(a_{i}, s_{\theta}(\bar{\nu}, v)\right)$,

- For each $k$ and $a_{i} \in \cup_{i \in I} A_{i}^{+}, x\left(a_{i}, s_{c}^{\theta}(\bar{\nu}, \nu, v)\right)=z_{k}^{\prime}(\nu, v)$ if and only if $x\left(a_{i}, s^{\theta}(\bar{\nu}, v)\right)=$ $z_{k}(v)$.

We prove that $s_{c}^{\theta}(\bar{\nu}, \nu, v)$ satisfies the required properties. Fix some $a_{i} \in \cup_{i \in I} A_{i}^{+}$. Point 1 is easily checked. Regarding Point 2, notice that since $\nu$ and $\bar{\nu}$ are smaller than $\min \left(\lambda^{\star}, \bar{\nu}^{\star}\right)$, $\mathbb{P}_{\nu}^{v}\left(x_{-i} \leq x\left(a_{-i}, s_{c}^{\theta}(\bar{\nu}, \nu, v)\right) \mid x\left(a_{i}, s_{c}^{\theta}(\bar{\nu}, \nu, v)\right)\right)=0=\mathbb{P}_{\bar{\nu}}^{v}\left(x_{-i} \leq x\left(a_{-i}, s^{\theta}(\bar{\nu}, v)\right) \mid x\left(a_{i}, s^{\theta}(\bar{\nu}, v)\right)\right)$, for all $a_{-i} \in A_{-i}^{-}$. Now, assume that $a_{-i} \in A_{-i}^{+}$. On the one hand, note that $\mid x\left(a_{j}, s_{c}^{\theta}(\bar{\nu}, \nu, v)\right)-$ $x\left(a_{i}, s_{c}^{\theta}(\bar{\nu}, \nu, v)\right) \mid \geq \nu$ if and only if $\left|x\left(a_{j}, s^{\theta}(\bar{\nu}, v)\right)-x\left(a_{i}, s^{\theta}(\bar{\nu}, v)\right)\right| \geq \bar{\nu}$. Moreover, if $\left|x\left(a_{j}, s_{c}^{\theta}(\bar{\nu}, \nu, v)\right)-x\left(a_{i}, s_{c}^{\theta}(\bar{\nu}, \nu, v)\right)\right|<\nu$, then:

$$
\frac{x\left(a_{j}, s_{c}^{\theta}(\bar{\nu}, \nu, v)\right)-x\left(a_{i}, s_{c}^{\theta}(\bar{\nu}, \nu, v)\right)}{\nu}=\frac{x\left(a_{j}, s^{\theta}(\bar{\nu}, v)\right)-x\left(a_{i}, s^{\theta}(\bar{\nu}, v)\right)}{\bar{\nu}} .
$$

On the other hand, since $\bar{\nu}<L$, we must have by the definition of dominance regions, $x\left(a_{i}, s^{\theta}(\bar{\nu}, v)\right) \in[\bar{\nu}, 1-\bar{\nu}]$ for all $a_{i} \neq \max A_{i}$, which by construction implies: $x\left(a_{i}, s_{c}^{\theta}(\bar{\nu}, \nu, v)\right) \in$ $[\nu, 1-\nu]$. Hence, Remark 1 below, which directly follows from the fact that the prior is uniformly distributed on $[0,1]$ in the one-dimensional global game $G_{\star}^{\theta}(\nu, v)$ allows to conclude the proof.

Remark 1. For each $\nu>0, x^{\star} \in[\nu, 1-\nu]^{|I|}$ and $v \in V$,

$$
\mathbb{P}_{\nu}^{v}\left(x_{-i} \leq x_{-i}^{\star} \mid x_{i}^{\star}\right)=\int_{-\frac{1}{2}}^{\frac{1}{2}} \prod_{j \neq i} \Phi_{j}^{v}\left(\frac{x_{j}^{\star}-x_{i}^{\star}}{\nu}+\eta_{i}\right) \phi_{i}^{v}\left(\eta_{i}\right) d \eta_{i} .
$$

Proof. It suffices to notice that since $x_{i}^{\star} \in[\nu, 1-\nu]$ and the prior is uniformly distributed on $[0,1]$, applying Bayes' rule:

$$
\mathbb{P}_{\nu}^{v}\left(x_{-i} \leq x_{-i}^{\star} \mid x_{i}^{\star}\right)=\frac{\int_{x_{i}^{\star}-\frac{\nu}{2}}^{x^{\star}+\frac{\nu}{2}} \prod_{j \neq i} \Phi_{j}^{v}\left(\frac{x_{j}^{\star}-t}{\nu}\right) \phi_{i}^{v}\left(\frac{x_{i}^{\star}-t}{v}\right) d t}{\int_{x_{i}^{\star}-\frac{\nu}{2}}^{x_{i}^{\star}+\frac{\nu}{2}} \phi_{i}^{v}\left(\frac{x_{i}^{\star}-t}{v}\right) d t} .
$$

Since $\int_{-\frac{1}{2}}^{\frac{1}{2}} \phi_{i}^{v}(l) d l=1$, the change in variable $\eta_{i}=\frac{x_{i}^{\star}-t}{\nu}$ yields the desired result.

For all $\lambda \in[-1,1]$ and $\nu>0$, define the strategy profile $s(\lambda, \bar{\nu}, \nu)$ in the $N$-dimensional global game $G_{\nu}(u, \phi, \psi)$ as follows for each player $i$ and signal $x_{i} \in \mathbb{R}^{N}$ : 
- For each action $a_{i} \in A_{i}^{+} \backslash \max A_{i}$, we have: $a_{i}^{\star} \leq s_{i}(\lambda, \bar{\nu}, \nu)\left(x_{i}\right) \leq a_{i}$ if and only if there exists $\tilde{t} \in\left[0, \tilde{t}^{\star}+\lambda\right]$ such that $\left\|\theta(\tilde{t})-x_{i}\right\| \leq \sqrt{\nu}+x\left(a_{i}, s_{c}^{\theta}(\bar{\nu}, \nu, \tilde{v})\right)-\tilde{t}^{\star}$ with $\tilde{v}=\frac{x_{i}-\theta(\tilde{t})}{\left\|x_{i}-\theta(\tilde{t})\right\|}$

- Otherwise, $s_{i}(\lambda, \bar{\nu}, \nu)\left(x_{i}\right)=\max A_{i}$.

We consider, for each $\nu>0$, the following sequence of strategy profiles $\left\{\bar{s}(\nu)^{n}\right\}_{n \in N}$. Let $\bar{s}(\nu)^{0}$ be the constant profile in which all players play $\max A_{i}$ for any signal. For each $n>0$, we set $\bar{s}(\nu)^{n}=s\left(\lambda^{n}(\nu), \bar{\nu}, \nu\right)$, where the sequence $\left\{\lambda^{n}(\nu)\right\}_{n \in N^{\star}}$ is defined recursively by :

$$
\lambda^{n+1}(\nu)=\sup \left\{\lambda \in[-1,1] \mid s_{i}(\lambda, \bar{\nu}, \nu) \geq \max \operatorname{BR}_{i}\left(\bar{s}_{-i}(\nu)^{n}\right), \forall i\right\}
$$

where $\max B R_{i}$ denotes the maximal best-response strategy of player $i$ to $\bar{s}_{-i}(\nu)^{n}$.

Lemma 2. There exists $\nu^{\star}>0$ such that for each $\nu<\nu^{\star}$, the sequence $\left\{\bar{s}(\nu)^{n}\right\}_{n \in N}$ is decreasing and each strategy profile $s_{\nu}$ surviving iterative strict dominance in the game $G_{\nu}(u, \phi, \psi)$ satisfies: $s_{\nu} \leq \bar{s}(\nu)^{n}$, for all $n \in \mathbb{N}$.

Proof of Lemma 2. There exists $\nu^{\star}$ such that for each $\nu<\nu^{\star}$ :

$$
\bar{\mu}-\frac{\nu}{2}>\sqrt{\nu}+C \nu
$$

(where $\bar{\mu}$ is as defined in Assumption 1.) Note that Equation (1) means that for each player $i$ and signal $x_{i} \notin B^{\bar{\mu}-\frac{\nu}{2}}(\Theta)$, we must have $\bar{s}_{i}^{n}\left(x_{i}\right)=\max A_{i}$, for all $n$. Now fix some $\nu<\nu^{\star}$. We prove Lemma 2 by induction. It is trivially true at rank 0 . Assume that it is also true at rank $n$, that is, assume that $\bar{s}(\nu)^{n+1} \leq \bar{s}(\nu)^{n}$ and that each strategy profile $s_{\nu}$ surviving iterative strict dominance satisfies: $s_{\nu} \leq \bar{s}(\nu)^{n}$. For each player $i$ and signal $x_{i} \in \mathbb{R}^{N}$, we need to distinguish two cases. If $x_{i} \notin B^{\bar{\mu}-\frac{\nu}{2}}(\Theta)$, we know by Equation (1) that $\bar{s}_{i}^{n+1}\left(x_{i}\right)=\max A_{i}$ and the result trivially follows. Now assume that $x_{i} \in B^{\bar{\mu}-\frac{\nu}{2}}(\Theta)$. Then by the triangle inequality, for each $t \in B^{\frac{\nu}{2}}\left(x_{i}\right), t \in B^{\bar{\mu}}(\Theta)$. This means that for each state parameter $t \in B^{\frac{\nu}{2}}\left(x_{i}\right)$, the payoff function associated with $t$ is supermodular. Consequently, on the one hand, by the second induction hypothesis, we deduce that each strategy $s_{\nu, i}$ surviving iterative strict dominance must satisfy: $s_{\nu, i}\left(x_{i}\right) \leq \max \mathrm{BR}_{i}\left(\bar{s}(\nu)^{n}\right)\left(x_{i}\right) \leq \bar{s}_{i}(\nu)^{n+1}\left(x_{i}\right)$. On the other hand, by the first induction hypothesis, we have: $\max \mathrm{BR}_{i}\left(\bar{s}(\nu)^{n+1}\right)\left(x_{i}\right) \leq \max _{\mathrm{BR}_{i}}\left(\bar{s}(\nu)^{n}\right)\left(x_{i}\right)$, which implies that $\lambda^{n+2}(\nu) \geq \lambda^{n+1}(\nu)$ and $\bar{s}(\nu)^{n+2} \leq \bar{s}(\nu)^{n+1}$.

The sequence $\left\{\lambda^{n}(\nu)\right\}_{n \in N}$ is increasing and bounded. Hence, the sequences $\left\{\lambda^{n}\right\}_{n \in N}$ and $\left\{\bar{s}(\nu)^{n}\right\}_{n \in N}$ converge and we note respectively $\bar{\lambda}$ and $\bar{s}(\nu)$ for their limits. By Lemma 2, for 
each $\nu$, each strategy profile $s_{\nu}$ surviving iterative strict dominance in the game $G_{\nu}(u, \phi, \psi)$ satisfies: $s_{\nu} \leq \bar{s}(\nu)$. Hence, to conclude the proof of Theorem 3, it suffices to establish that there exists $\nu^{\star}>0$ such that for each $\nu<\nu^{\star}, \bar{\lambda}(\nu) \geq 0$. (Indeed, since $x\left(a_{i}^{\star}, s_{c}^{\theta}(\bar{\nu}, \nu, \tilde{v})\right)-\tilde{t}^{\star} \geq$ 0 , the above condition implies that $\bar{s}(\nu)\left(x_{i}\right)=a_{i}^{\star}$ for all $x_{i} \in \mathbb{R}^{N}$ with $\left\|t^{\star}-x_{i}\right\| \leq \sqrt{\nu}$.) Proposition 2 below is the main step of the proof.

Proposition 2. For any given $\delta>0$, there exists $\nu(\delta)$ such that the following property is satisfied for all $\lambda \in[-1,1]$ and $\nu<\nu(\delta)$. For each player $i$, action $a_{i} \in A_{i}^{+} \backslash \max A_{i}$ and signal $x_{i} \in R^{N}$ with $s_{i}(\lambda, \bar{\nu}, \nu)\left(x_{i}\right) \leq a_{i}$, there exists a unit vector $\tilde{v} \in V$ such that:

$$
\mathbb{P}_{\nu}\left(s_{-i}(\lambda, \bar{\nu}, v)\left(x_{-i}\right) \leq a_{-i} \mid x_{i}\right) \geq \mathbb{P}_{\bar{\nu}}^{\tilde{v}}\left(s_{-i}^{\theta}(\bar{\nu}, \tilde{v})\left(x_{-i}\right) \leq a_{-i} \mid x\left(a_{i}, s^{\theta}(\bar{\nu}, \tilde{v})\right)\right)-\delta,
$$

for all $a_{-i} \in A_{-i}$.

Proof. See Appendix.

The proof of Proposition 3 below comes from the strict monotonicity of the payoff function $\left(u_{i}^{\theta}\right)_{i \in I}$.

Proposition 3. There exists $\nu^{\star}>0$ such that the following property is satisfied for all $\lambda \leq 0$ and $\nu<\nu^{\star}$. For each player $i$, action $a_{i} \in A_{i}^{+} \backslash \max A_{i}$, unit vector $v \in V$ and signal $x_{i} \in \mathbb{R}^{N}$ with $s(\lambda, \bar{\nu}, \nu)\left(x_{i}\right) \leq a_{i}$ :

$$
\Delta u_{i}\left(a_{i}^{\prime} \rightarrow a_{i}, a_{-i}, t\right) \geq \Delta u_{i}^{\theta}\left(a_{i}^{\prime} \rightarrow a_{i}, a_{-i}, x\left(a_{i}, s^{\theta}(\bar{\nu}, v)\right)\right)+\frac{\lambda^{\star} K}{2},
$$

for all $a_{i}^{\prime} \in A_{i}^{+}$with $a_{i}^{\prime}>a_{i}, a_{-i} \in A_{-i}$ and $t \in B^{\frac{\nu}{2}}\left(x_{i}\right)$.

Proof. See Appendix.

We are now in a position to conclude the proof of Theorem 3. Since $s^{\theta}(\bar{\nu}, \tilde{v})$ is an equilibrium profile of the one-dimensional (simplified) global game $G_{\star}^{\theta}(\bar{\nu}, \tilde{v})$,

$$
E_{\bar{\nu}}^{\tilde{v}}\left[\Delta u_{i}^{\theta}\left(a_{i}^{\prime} \rightarrow a_{i}\right) \mid x\left(a_{i}, s^{\theta}(\bar{\nu}, \tilde{v})\right), s_{-i}^{\theta}(\bar{\nu}, \tilde{v})\right] \geq 0
$$

for all $a_{i}^{\prime} \in A_{i}$. Combining Propositions 2 and 3, we know by strategic complementarities that there exists $\nu^{\star}>0$ such that the following property holds for all $\lambda<0$ and $\nu<\nu^{\star}$. For each player $i$, action $a_{i} \in A_{i}^{+} \backslash \max A_{i}$ and $x_{i} \in \mathbb{R}^{N}$ with $s_{i}(\lambda, \bar{\nu}, \nu)\left(x_{i}\right) \leq a_{i}$, there exists a unit vector $\tilde{v} \in V$ such that:

$$
E_{\nu}\left[\Delta u_{i}\left(a_{i}^{\prime} \rightarrow a_{i}\right) \mid x_{i}, s_{-i}(\lambda, \bar{\nu}, \nu)\right] \geq E_{\bar{\nu}}^{\tilde{v}}\left[\Delta u_{i}^{\theta}\left(a_{i}^{\prime} \rightarrow a_{i}\right) \mid x\left(a_{i}, s^{\theta}(\bar{\nu}, \tilde{v})\right), s_{-i}^{\theta}(\bar{\nu}, \tilde{v})\right]+\frac{\lambda^{\star} K}{4},
$$


for all $a_{i}^{\prime} \in A_{i}^{+}$with $a_{i}^{\prime}>a_{i}$.

Now, fix some $\lambda<0$ and $\nu<\nu^{\star}$ such that $\sqrt{\nu}+C \nu \leq \bar{\mu}$. For each player $i$, action $a_{i} \in A_{i}^{+} \backslash \max A_{i}$ and $x_{i} \in \mathbb{R}^{N}$ with $s_{i}(\lambda, \bar{\nu}, \nu)\left(x_{i}\right) \leq a_{i}$, Equations (3) and (4) together imply:

$$
E_{\nu}\left[\Delta u_{i}\left(a_{i}^{\prime} \rightarrow a_{i}\right) \mid x_{i}, s_{-i}(\lambda, \bar{\nu}, \nu)\right] \geq \frac{\lambda^{\star} K}{4},
$$

for all $a_{i}^{\prime} \in A_{i}^{+}$with $a_{i}^{\prime}>a_{i}$. Notice on the one hand that there exists a function $f(\lambda, \nu)$ : $\mathbb{R}_{+}^{\star} \rightarrow \mathbb{R}_{+}^{\star}$ such that for each $\varepsilon>0, i \in I, a_{i} \in A_{i}^{+} \backslash \max A_{i}$ and $x_{i}^{\prime} \in \mathbb{R}^{N}$ with $s_{i}(\lambda+$ $f(\lambda, \nu)(\varepsilon), \bar{\nu}, \nu)\left(x_{i}\right) \leq a_{i}$, there exists $x_{i} \in \mathbb{R}^{N}$ such that: (i) $s_{i}(\lambda, \bar{\nu}, \nu)\left(x_{i}\right) \leq a_{i}$ and (ii) $\left\|x_{i}-x_{i}^{\prime}\right\|<\varepsilon$. On the other hand, since the function $x_{i} \mapsto E_{\nu}\left[\Delta u_{i}\left(a_{i}^{\prime} \rightarrow a_{i}\right) \mid x_{i}, s(\lambda, \bar{\nu}, \nu)\right]$ is continuous and the set of $x_{i} \in \mathbb{R}^{N}$ such that $s(\lambda, \bar{\nu}, \nu)\left(x_{i}\right) \leq a_{i}$ for some $a_{i}<\max A_{i}$ is included in the compact set $B^{\bar{\mu}}(\Theta)$, there exists $\varepsilon>0$ such that :

$$
\left|E_{\nu}\left[\Delta u_{i}\left(a_{i}^{\prime} \rightarrow a_{i}\right) \mid x_{i}, s_{-i}(\lambda, \bar{\nu}, \nu)\right]-E_{\nu}\left[\Delta u_{i}\left(a_{i}^{\prime} \rightarrow a_{i}\right) \mid x_{i}^{\prime}, s_{-i}(\lambda, \bar{\nu}, \nu)\right]\right| \leq \frac{\lambda^{\star} K}{8},
$$

for all $x_{i}, x_{i}^{\prime} \in \mathbb{R}^{N}$ with $s(\lambda, \bar{\nu}, \nu)\left(x_{i}\right) \leq a_{i}$ for some $a_{i}<\max A_{i}$ and $\left\|x_{i}-x_{i}^{\prime}\right\|<\varepsilon$. The two above arguments together yield that, by Equation (5), there must exist some $\tau(\lambda, \nu)>0$ such that for each $i, a_{i} \in A_{i}^{+} \backslash \max A_{i}$ and $x_{i}^{\prime} \in \mathbb{R}^{N}$ with $s_{i}(\lambda+\tau(\lambda, \nu), \bar{\nu}, \nu)\left(x_{i}^{\prime}\right) \leq a_{i}$,

$$
E_{\nu}\left[\Delta u_{i}\left(a_{i}^{\prime} \rightarrow a_{i}\right) \mid x_{i}^{\prime}, s_{-i}(\lambda, \bar{\nu}, \nu)\right] \geq \frac{\lambda^{\star} K}{8}
$$

for all $a_{i}^{\prime} \in A_{i}$ with $a_{i}^{\prime}>a_{i}$. Consequently, $\max B R(s(\lambda, \bar{\nu}, \nu)) \leq s(\lambda+\tau(\lambda, \nu), \bar{\nu}, \nu)$, which implies that $\lambda$ cannot be equal to $\bar{\lambda}(\nu)$. This establishes that $\bar{\lambda}(\nu)$ must be positive and thus concludes the proof.

\section{References}

[1] Basteck C., Daniels T, Heinemann F., 2011. "Characterizing Equilibrium Selection in Global Games with Strategic Complementarities," mimeo.

[2] Carlsson, H., Van Damme, E., 1993. "Global games and Equilibrium selection", Econometrica, 61, 989-1018.

[3] Frankel, D., Morris, S., Pauzner, A., 2003. "Equilibrium Selection in Global Games with Strategic Complementarities," J. Econ. Theory, 108,1-44. 
[4] Harsanyi, J. and R. Selten, 1988. "A General Theory of Equilibrium Selection in Games", Cambridge, MIT Press.

[5] Kajii, A., Morris, S., 1997. "The Robustness of Equilibria to Incomplete Information," Econometrica, 65,1283-1309.

[6] Morris, S., 2008. "Global Games", The New Palgrave Dictionary of Economics, Steven Durlauf and Lawrence Blume, Eds. Palgrave Macmillan.

[7] Morris, S., Shin, H.S., 2003. "Global Games: Theory and Applications" Advances in Economics and Econometrics (Proceedings of the Eighth World Congress of the Econometric Society), edited by M. Dewatripont, L.Hansen and S. Turnovsky; Cambridge University Press.

[8] Morris, S., Ui, T., 2003. "Generalized Potentials and Robust Sets of Equilibria", J. Econ. Theory, 124, 45-78.

[9] Oyama, D., Tercieux, O., 2009. "Iterated Potentials and the Robustness of Equilibria", Journal of Economic Theory, 144, 1726-1769

[10] Oyama, D., Takahashi, S., 2011 "On the Relationship between Robustness to Incomplete Information and Noise-Independent Selection in Global Games" Forthcoming in Journal of Mathematical Economics. 


\section{Appendix}

\subsection{Proof of Proposition 1.}

Recall that, given a real non-negative parameter $\varepsilon$, a strategy profile is said to be an $\varepsilon$ equilibrium if it is not possible for any player to gain more than $\varepsilon$ in expected payoff by unilaterally deviating from his strategy. For all increasing and right-continuous strategy profiles $s$ and $s^{\prime}$ in a one-dimensional global game $G_{\star}^{\theta}(\nu, v)$, we define:

$$
\Delta\left(s, s^{\prime}\right)=\max _{i \in I} \max _{a_{i} \in A_{i}}\left|x\left(a_{i}, s\right)-x\left(a_{i}, s^{\prime}\right)\right|,
$$

In addition, for each $i$ and $a_{i} \in A_{i} \backslash \max A_{i}$, we set:

$$
\left(a_{i}, s\right)^{+}=\min \left(a_{i}^{\prime} \in A_{i} \mid \exists x_{i}>x\left(a_{i}, s\right) \text { with } s_{i}\left(x_{i}\right)=a_{i}\right),
$$

and,

$$
\left(a_{i}, s\right)^{-}=\max \left(a_{i}^{\prime} \in A_{i} \mid \exists x_{i}<x\left(a_{i}, s\right) \text { with } s_{i}\left(x_{i}\right)=a_{i}\right),
$$

Lemma 3. For each unit vector $v \in V, \nu>0, \varepsilon \geq 0$ and $\varepsilon$-equilibria in increasing strategies $s$ and $s^{\prime}$ in $G_{\star}^{\theta}(\nu, v): \Delta\left(s, s^{\prime}\right) \leq \frac{2 \varepsilon}{K}$.

Proof of Lemma 3. Assume, w.l.o.g., that there exists a player $i$ and an action $\hat{a}_{i} \in A_{i}$ such that $\Delta\left(s, s^{\prime}\right)=x\left(\hat{a}_{i}, s^{\prime}\right)-x\left(\hat{a}_{i}, s\right)>0$. Let $s^{\prime \prime}=\left\{s_{i}^{\prime \prime}\right\}_{i \in I}$ be defined for each player $i$ by: $s_{i}^{\prime \prime}\left(x_{i}\right)=s_{i}\left(x_{i}-\Delta\left(s, s^{\prime}\right)\right)$ for each $x_{i} \geq-\frac{\nu}{2}+\Delta\left(s, s^{\prime}\right)$ and $s_{i}^{\prime \prime}\left(x_{i}\right)=\min A_{i}$ otherwise. Notice that $x\left(\hat{a}_{i}, s^{\prime}\right)=x\left(\hat{a}_{i}, s^{\prime \prime}\right)$ and $\left(\hat{a}_{i}, s^{\prime}\right)^{-} \leq \hat{a}_{i}<\left(\hat{a}_{i}, s\right)^{+}$. Hence, since $s^{\prime} \geq s^{\prime \prime}$, strategic complementarities imply that:

$$
\mathbf{E}_{\nu}^{v}\left[\Delta u_{i}^{\theta}\left(\left(\hat{a}_{i}, s^{\prime}\right)^{-} \rightarrow\left(\hat{a}_{i}, s\right)^{+}\right) \mid x\left(\hat{a}_{i}, s^{\prime}\right), s_{-i}^{\prime}\right] \geq \mathbf{E}_{\nu}^{v}\left[\Delta u_{i}^{\theta}\left(\left(\hat{a}_{i}, s^{\prime}\right)^{-} \rightarrow\left(\hat{a}_{i}, s\right)^{+}\right) \mid x\left(\hat{a}_{i}, s^{\prime \prime}\right), s_{-i}^{\prime \prime}\right] .
$$

Now, on the one hand, since $s$ is an $\varepsilon$-equilibrium in $G_{\star}^{\theta}(\nu, v)$,

$$
\mathbf{E}_{\nu}^{v}\left[\Delta u_{i}^{\theta}\left(\left(\hat{a}_{i}, s^{\prime}\right)^{-} \rightarrow\left(\hat{a}_{i}, s\right)^{+}\right) \mid x\left(\hat{a}_{i}, s\right), s\right] \geq-\varepsilon .
$$

Consequently, using the fact that the prior is uniformly distributed on $[0,1]$ and strict monotonicity,

$$
\mathbf{E}_{\nu}^{v}\left[\Delta u_{i}^{\theta}\left(\left(\hat{a}_{i}, s^{\prime}\right)^{-} \rightarrow\left(\hat{a}_{i}, s\right)^{+}\right) \mid x\left(\hat{a}_{i}, s^{\prime \prime}\right), s_{-i}^{\prime \prime}\right] \geq-\varepsilon+\Delta\left(s, s^{\prime}\right) K .
$$

On the other hand, since $s^{\prime}$ is an $\varepsilon$-equilibrium, we have:

$$
\mathbf{E}_{\nu}^{v}\left[\Delta u_{i}^{\theta}\left(\left(\hat{a}_{i}, s\right)^{+} \rightarrow\left(\hat{a}_{i}, s^{\prime}\right)^{-}\right) \mid x\left(\hat{a}_{i}, s^{\prime}\right), s^{\prime}\right] \geq-\varepsilon .
$$


Hence, Equations (6) and (7) together yield: $\Delta\left(s, s^{\prime}\right) \leq \frac{2 \varepsilon}{K}$, concluding the proof of Lemma 3.

Lemma 4. For any $\varepsilon>0$, there exists $\nu^{\star}>0$ such that for each unit vector $v \in V$ and $\nu, \nu^{\prime}>$ 0 with $\nu^{\prime}<\nu<\nu^{\star}$, there exists an increasing profile $s_{\nu \rightarrow \nu^{\prime}}^{v}$ such that 1) $\Delta\left(s_{\nu \rightarrow \nu^{\prime}}^{v}, s^{\theta}\left(\nu^{\prime}, v\right)\right)<\varepsilon$ and 2) $\Delta\left(s_{\nu \rightarrow \nu^{\prime}}^{v}, s^{\theta}(\nu, v)\right)<\varepsilon$.

Proof of Lemma 4. Fix some $\varepsilon>0$. We first need to introduce some additional notations. For each $\nu>0$ and unit vector $v$, we define the equivalence relation $\sim_{\nu}^{v}$ as follows. For each $a_{i}, a_{j} \in \cup_{i \in I} A_{i}, a_{i} \sim_{\nu}^{v} a_{j}$ if and only if there exists a sequence of actions $\left\{a^{r}\right\}_{r=1, \ldots, R} \in \cup_{i \in I} A_{i}$ such that: $a^{1}=a_{i}, a^{R}=a_{j}$, and, for all $r=1, \ldots, R-1$, $\left|x\left(a^{r}, s^{\theta}(\nu, v)\right)-x\left(a^{r+1}, s^{\theta}(\nu, v)\right)\right| \leq \nu$. We also define the order $\preceq$ on the quotient space induced by $\sim_{\nu}^{v}$ as follows. For all actions $a_{i}, a_{j} \in \cup_{i \in I} A_{i}, \quad\left[a_{i}\right]_{\nu}^{v} \preceq\left[a_{j}\right]_{\nu}^{v}$ if and only if $x\left(a_{k}, s^{\theta}(\nu, v)\right) \leq x\left(a_{l}, s^{\theta}(\nu, v)\right)$ for all actions $a_{k}, a_{l} \in \cup_{i \in I} A_{i}$ with $\left[a_{k}\right]_{\nu}^{v}=\left[a_{i}\right]_{\nu}^{v}$ and $\left[a_{l}\right]_{\nu}^{v}=$ $\left[a_{j}\right]_{\nu}^{v}$. It can easily be checked that $\preceq$ define a total order on the quotient space. Now, for each action $a_{i} \in \cup_{i \in I} A_{i}$, define $x\left(\left[a_{i}\right]_{\nu}^{v}\right)$ and $\delta_{\nu}^{v}\left(a_{i}\right)$ by:

$$
x\left(\left[a_{i}\right]_{\nu}^{v}\right)=\min _{a_{j} \in\left[a_{i}\right]_{\nu}^{v}}\left\{x\left(a_{j}, s^{\theta}(\nu, v)\right)\right\}
$$

and,

$$
\delta_{\nu}^{v}\left(a_{i}\right)=\left(x\left(a_{i}, s^{\theta}(\nu, v)\right)-x\left(\left[a_{i}\right]_{\nu}^{v}\right)\right) / \nu
$$

Finally, let the increasing strategy profile $s_{\nu \rightarrow \nu^{\prime}}^{v}$ be such that for each player $i$ and action $a_{i} \in A_{i}$,

$$
x\left(a_{i}, s_{\nu \rightarrow \nu^{\prime}}^{v}\right)=x\left(\left[a_{i}\right]_{\nu}^{v}\right)+\nu^{\prime} \delta_{\nu}^{v}\left(a_{i}\right) .
$$

Now, fix some $\nu<L$. Remark 2 below follows from the construction of $s_{\nu \rightarrow \nu^{\prime}}^{v}$ and the fact that the prior is uniformly distributed on the interval $[0,1]$ in $G_{\star}^{\theta}(\nu, v)$.

Remark 2. For each player $i$ and each action $a_{i} \in A_{i} \backslash \max A_{i}$,

$$
\mathbb{P}_{\nu}^{v}\left(x_{-i} \leq x\left(a_{-i}, s^{\theta}(\nu, v)\right) \mid x\left(a_{i}, s^{\theta}(\nu, v)\right)\right)=\mathbb{P}_{\nu^{\prime}}^{v}\left(x_{-i} \leq x\left(a_{-i}, s_{\nu \rightarrow \nu^{\prime}}^{v}\right) \mid x\left(a_{i}, s_{\nu \rightarrow \nu^{\prime}}^{v}\right)\right)
$$

for all action profiles $a_{-i} \in A_{-i}$.

Proof. Since $s^{\theta}(\nu, v)$ is an equilibrium profile in the simplified game $G_{\star}^{\theta}\left(\nu^{\prime}, v\right)$, the existence of dominance regions yields: $x\left(a_{i}, s^{\theta}(\nu, v)\right) \in[L, 1-L] \subset[\nu, 1-\nu]$, for each 
$a_{i} \in A_{i} \backslash \max A_{i}$ which in turn implies : $x\left(a_{i}, s_{\nu \rightarrow \nu^{\prime}}^{v}\right) \in\left[\nu^{\prime}, 1-\nu^{\prime}\right]$, for all $\nu^{\prime}<\nu$. Note that if $a_{i} \prec a_{j}$, then

$$
\mathbb{P}_{\nu}^{v}\left(x_{j} \leq x\left(a_{j}, s^{\theta}(\nu, v)\right) \mid x\left(a_{i}, s^{\theta}(\nu, v)\right)\right)=1=\mathbb{P}_{\nu^{\prime}}^{v}\left(x_{j} \leq x\left(a_{j}, s_{\nu \rightarrow \nu^{\prime}}^{v}\right) \mid x\left(a_{i}, s_{\nu \rightarrow \nu^{\prime}}^{v}\right)\right)
$$

And, symmetrically,

$$
\mathbb{P}_{\nu}^{v}\left(x_{i} \leq x\left(a_{i}, s^{\theta}(\nu, v)\right) \mid x\left(a_{j}, s^{\theta}(\nu, v)\right)\right)=0=\mathbb{P}_{\nu^{\prime}}^{v}\left(x_{i} \leq x\left(a_{i}, s_{\nu \rightarrow \nu^{\prime}}^{v}\right) \mid x\left(a_{j}, s_{\nu \rightarrow \nu^{\prime}}^{v}\right)\right)
$$

In addition, for each $a_{i}, a_{j} \in \cup_{i \in I} A_{i}$ with $a_{i} \sim_{\nu}^{v} a_{j}$,

$$
\frac{x\left(a_{i}, s^{\theta}(\nu, v)\right)-x\left(a_{j}, s^{\theta}(\nu, v)\right)}{\nu}=\frac{x\left(a_{i}, s_{\nu \rightarrow \nu^{\prime}}^{v}\right)-x\left(a_{j}, s_{\nu \rightarrow \nu^{\prime}}^{v}\right)}{\nu^{\prime}} .
$$

Hence, Remark 1 allows to conclude the proof.

We now prove that the strategy profile $s_{\nu \rightarrow \nu^{\prime}}^{v}$ satisfies the required properties. Notice that for all actions $a_{i}, a_{j} \in \cup_{i \in I} A_{i}$ with $\left[a_{i}\right]_{\nu}^{v}=\left[a_{j}\right]_{\nu}^{v}$, we have: $\left|x\left(a_{i}, s^{\theta}(\nu, v)\right)-x\left(a_{j}, s^{\theta}(\nu, v)\right)\right| \leq C \nu$ (where we recall that $C$ denotes the cardinal of the set $\cup_{i \in I} A_{i}$ ). Consequently, for each $a_{i} \in \cup_{i \in I} A_{i}, \delta_{\nu}^{v}\left(a_{i}\right) \in[0, C]$. Hence, for each $\nu^{\prime}<\nu<\frac{\varepsilon}{C}$, by construction of the strategy profile $s_{\nu \rightarrow \nu^{\prime}}^{v}$,

$$
\begin{aligned}
\left|x\left(a_{i}, s^{\theta}(\nu, v)\right)-x\left(a_{i}, s_{\nu \rightarrow \nu^{\prime}}^{v}\right)\right|=\left|x\left(\left[a_{i}\right]_{\nu}^{v}\right)+\nu \delta_{\nu}^{v}\left(a_{i}\right)-\left(x\left(\left[a_{i}\right]_{\nu}^{v}\right)+\nu^{\prime} \delta_{\nu}^{v}\left(a_{i}\right)\right)\right| & \leq\left(\nu-\nu^{\prime}\right) \delta_{\nu}^{v}\left(a_{i}\right) \\
& \leq C \nu<\varepsilon, \quad(9)
\end{aligned}
$$

for each player $i$ and each action $a_{i} \in A_{i}$, which establishes Point 2 of Lemma 4. Let us turn to Point 1. We show that for each $\varepsilon>0$, there exists $\nu>0$ such that $s_{\nu \rightarrow \nu^{\prime}}^{v}$ is an $\varepsilon$-equilibrium of $G_{\star}^{\theta}\left(\nu^{\prime}, v\right)$ for all $\nu^{\prime}<\nu$. Lemma 3 then allows to conclude the proof. In order to do so, by a standard argument, using strategic complementarities and the monotonicity of $u^{\theta}$, it suffices to establish that the following properties are satisfied for each $i$ and $a_{i} \in A_{i} \backslash \max A_{i}$,

$$
\mathbf{E}_{\nu^{\prime}}^{v}\left[\Delta u_{i}^{\theta}\left(a_{i}^{\prime} \rightarrow\left(a_{i}, s_{\nu \rightarrow \nu^{\prime}}^{v}\right)^{+}\right) \mid x\left(a_{i}, s_{\nu \rightarrow \nu^{\prime}}^{v}\right), s_{\nu \rightarrow \nu^{\prime},-i}^{v}\right] \geq-\varepsilon,
$$

and,

$$
\mathbf{E}_{\nu^{\prime}}^{v}\left[\Delta u_{i}^{\theta}\left(a_{i}^{\prime} \rightarrow\left(a_{i}, s_{\nu \rightarrow \nu^{\prime}}^{v}\right)^{-}\right) \mid x\left(a_{i}, s_{\nu \rightarrow \nu^{\prime}}^{v}\right), s_{\nu \rightarrow \nu^{\prime},-i}^{v}\right] \geq-\varepsilon,
$$

for all $a_{i}^{\prime} \in A_{i}$. On the one hand, since $s^{\theta}(\nu, v)$ is an equilibrium of the game $G_{\star}^{\theta}(\nu, v)$,

$$
\mathbf{E}_{\nu}^{v}\left[\Delta u_{i}^{\theta}\left(a_{i}^{\prime} \rightarrow\left(a_{i}, s^{\theta}(\nu, v)\right)^{+}\right) \mid x\left(a_{i}, s^{\theta}(\nu, v)\right), s_{-i}^{\theta}(\nu, v)\right] \geq 0
$$


and

$$
\mathbf{E}_{\nu^{\prime}}^{v}\left[\Delta u_{i}^{\theta}\left(a_{i}^{\prime} \rightarrow\left(a_{i}, s^{\theta}(\nu, v)^{-}\right) \mid x\left(a_{i}, s^{\theta}(\nu, v)\right), s_{-i}^{\theta}(\nu, v)\right] \geq 0\right.
$$

for all actions $a_{i}^{\prime} \in A_{i}$. On the other hand, recall that for each player $i$ and each action profile $a \in A$, the function $u_{i}^{\theta}(a, \tilde{t})$ is continuous in $\tilde{t}$. Consequently, since the interval $[0,1]$ is compact, there must exist a function $\xi^{\theta}: \mathbb{R}_{+}^{\star} \rightarrow \mathbb{R}_{+}^{\star}$ such that for all $i, a \in A, \varepsilon>0$ and $x_{i}, x_{i}^{\prime} \in[0,1]$ with $\left|x_{i}-x_{i}^{\prime}\right| \leq \xi^{\theta}(\varepsilon):\left|u_{i}\left(a, x_{i}\right)-u_{i}\left(a, x_{i}^{\prime}\right)\right| \leq \varepsilon$. Now, choose $\nu^{\prime}<\nu<\frac{\xi^{\theta}\left(\frac{\varepsilon}{2}\right)}{C}$. By Equation (9) above, we must have: $\left|x\left(a_{i}, s_{\nu \rightarrow \nu^{\prime}}^{v}\right)-x\left(a_{i}, s^{\theta}(\nu, v)\right)\right| \leq \xi^{\theta}\left(\frac{\varepsilon}{2}\right)$ which implies:

$$
\left|u_{i}^{\theta}\left(a, x\left(a_{i}, s_{\nu \rightarrow \nu^{\prime}}^{v}\right)\right)-u_{i}^{\theta}\left(a, x\left(a_{i}, s^{\theta}(\nu, v)\right)\right)\right| \leq \frac{\varepsilon}{2},
$$

for all $a \in A$. And,

$$
\left|\Delta u_{i}^{\theta}\left(a_{i} \rightarrow a_{i}^{\prime}, a_{-i}, x\left(a_{i}, s^{\nu \rightarrow \nu^{\prime}}\right)\right)-\Delta u_{i}^{\theta}\left(a_{i} \rightarrow a_{i}^{\prime}, a_{-i}, x\left(a_{i}, s^{\theta}(\nu, v)\right)\right)\right| \leq \varepsilon
$$

for all $a_{-i} \in A_{-i}$ and $a_{i}, a_{i}^{\prime} \in A_{i}$. Consequently, Remark 2 above allows to conclude the proof.

We now conclude the proof of Proposition 1. For each unit vector $v \in V$, set $t\left(a_{i}, v\right)=$ $\sup \left(x_{i} \in \mathbb{R} \mid s_{\star}^{\theta}(v)\left(x_{i}\right) \leq a_{i}\right)$. By Lemma 4, for each $\varepsilon>0$, there exists $\nu^{\star}$ such that, for each unit vector $v$ and $\nu, \nu^{\prime}<\nu^{\star}, \Delta\left(s^{\theta}(\nu, v), s^{\theta}\left(\nu^{\prime}, v\right)\right) \leq \varepsilon$. Consequently, the sequence of functions $x\left(a_{i}, s^{\theta}(\nu, v)\right)$ converges uniformly in $v$ toward the function $t\left(a_{i}, v\right)$ as $\nu$ tends toward zero. In addition, notice that for each $d \in\left[-\frac{1}{2}, \frac{1}{2}\right]$, the function $v \mapsto \phi_{i}^{v}(d)$ is continuous. Applying Lemma 3, we deduce that the function $v \mapsto x\left(a_{i}, s^{\theta}(\bar{\nu}, v)\right)$ is also continuous. Since $x\left(a_{i}, s^{\theta}(\nu, v)\right)$ converges uniformly in $v$ toward the function $t\left(a_{i}, v\right)$, this finally yields that the function $v \mapsto t\left(a_{i}, v\right)$ is continuous. Since by assumption we have: $t\left(a_{i}, v\right)>\tilde{t}^{\star}$, for each $a_{i} \in A_{i}^{+}$and $v$, we conclude that there must exist $\lambda^{\star}>0$ and $\bar{\nu}^{\star}>0$ such that for each $\bar{\nu}<\bar{\nu}^{\star}, x\left(a_{i}, s^{\theta}(\bar{\nu}, v)\right) \geq \tilde{t}^{\star}+\lambda^{\star}$. A symmetric argument holds for each $a_{i} \in A_{i}^{-}$.

\subsection{Proof of Proposition 2.}

We first introduce some additional notations. For any signal $x_{i} \in \mathbb{R}^{N}$, unit vector $v \in V$ and $d_{j} \in[-1,1]$, we define $L_{\nu}\left(x_{i}, v, d_{j}\right)$ and $H_{\nu}\left(x_{i}, v, d_{j}\right)$ respectively by:

$$
L_{\nu}\left(x_{i}, v, d_{j}\right)=\left\{x_{j} \in \mathbb{R}^{N} \mid v \cdot\left(x_{j}-x_{i}\right)=\nu d_{j}\right\},
$$

and,

$$
H_{\nu}\left(x_{i}, v, d_{j}\right)=\left\{x_{j} \in \mathbb{R}^{N} \mid v \cdot\left(x_{j}-x_{i}\right) \leq \nu d_{j}\right\} .
$$


Similarly, for any $x_{i} \in \mathbb{R}^{N}, v \in \mathbb{R}^{N}$ and $d_{-i} \in[-1,1]^{|-1|}$, we define $L_{\nu}\left(x_{i}, v, d_{-i}\right)$ and $H_{\nu}\left(x_{i}, v, d_{-i}\right)$ respectively by: $L_{\nu}\left(x_{i}, v, d_{-i}\right)=\Pi_{j \neq i} L_{\nu}\left(x_{i}, v, d_{j}\right)$ and $H_{\nu}\left(x_{i}, v, d_{-i}\right)=\Pi_{j \neq i} H_{\nu}\left(x_{i}, v, d_{j}\right)$. For each $a_{i} \in \cup_{i \in I} A_{i}^{+}$and unit vector $v$, notice that $\frac{1}{\nu}\left(x\left(a_{i}, s_{c}^{\theta}(\nu, \bar{\nu}, v)\right)-\tilde{t}^{\star}\right)$ does not depend on $\nu$ and set:

$$
\kappa\left(a_{i}, \bar{\nu}, v\right)=\frac{1}{\nu}\left(x\left(a_{i}, s_{c}^{\theta}(\nu, \bar{\nu}, v)\right)-\tilde{t}^{\star}\right) .
$$

By a slight abuse of notation, we write $\kappa\left(a_{-i}, \bar{\nu}, v\right)$ for the vector $\left(\kappa\left(a_{j}, \bar{\nu}, v\right)\right)_{j \neq i}$ and $\kappa\left(a_{-i}, \bar{\nu}, v\right)-$ $\kappa\left(a_{i}, \bar{\nu}, v\right)$ for the vector $\left(\kappa\left(a_{j}, \bar{\nu}, v\right)-\kappa\left(a_{i}, \bar{\nu}, v\right)\right)_{j \neq i}$.

Lemma 5. For any $\delta>0$, there exists $\nu_{1}(\delta)>0$ such that for all $\nu<\nu_{1}(\delta), \lambda \in[-1,1]$, $i \in I, a_{i} \in A_{i}^{+} \backslash \max A_{i}$ and $x_{i} \in B^{\bar{\mu}-\frac{\nu}{2}}(\Theta)$ with $s_{i}(\lambda, \bar{\nu}, \nu)\left(x_{i}\right) \leq a_{i}$,

$\mathbb{P}_{\nu}\left(x_{-i} \in H_{\nu}\left(x_{i}, v, \kappa\left(a_{-i}, \bar{\nu}, v\right)-\kappa\left(a_{i}, \bar{\nu}, v\right)\right) \mid x_{i}\right) \geq \mathbb{P}_{\bar{\nu}}^{v}\left(s_{-i}^{\theta}(\bar{\nu}, v)\left(x_{-i}\right) \leq a_{-i} \mid x\left(a_{i}, s^{\theta}(\bar{\nu}, v)\right)\right)-\delta$ for all unit vectors $v \in V$ and $a_{-i} \in A_{-i}^{+}$.

Proof of Lemma 5. Fix some $\delta>0$. For each $x_{i} \in B^{\bar{\mu}-\frac{\nu}{2}}(\Theta)$, define $m_{\nu}\left(x_{i}\right)$ by

$$
m_{\nu}\left(x_{i}\right)=\frac{\min _{t \in B^{\frac{\nu}{2}}\left(x_{i}\right)} \psi(t)}{\max _{t \in B^{\frac{\nu}{2}}\left(x_{i}\right)} \psi(t)}
$$

The set $B^{\bar{\mu}}(\Theta)$ is compact and the prior density $\psi$ is continuous and strictly positive on $B^{\bar{\mu}}(\Theta)$. Hence, there exists $\nu(\delta)>0$ such that for each $\nu<\nu(\delta)$ and $x_{i} \in B^{\bar{\mu}-\frac{\nu}{2}}(\Theta)$, $m_{\nu}\left(x_{i}\right)>1-\delta$. Now assume that $\nu<\nu(\delta)$ and pick some $i, a_{i} \in A_{i}^{+} \backslash \max A_{i}, a_{-i} \in A_{-i}^{+}$ and $x_{i} \in B^{\bar{\mu}-\frac{\nu}{2}}(\Theta)$ with $s_{i}(\lambda, \bar{\nu}, \nu)\left(x_{i}\right) \leq a_{i}$. On the one hand, applying Bayes' rule,

$$
\mathbb{P}_{\nu}\left(t \in L_{\nu}\left(x_{i}, v, d\right) \mid x_{i}\right)=\frac{\int_{t \in L_{\nu}\left(x_{i}, v, d\right)} \phi_{i}^{v}\left(\frac{x_{i}-t}{v}\right) \psi(t) d t}{\int \phi_{i}^{v}\left(\frac{x_{i}-t}{v}\right) \psi(t) d t},
$$

for all unit vectors $v \in V$ and $d \in[-1,1]$. This implies:

$$
m^{\nu}\left(x_{i}\right) \phi_{i}^{v}(-d) \leq \mathbb{P}_{\nu}\left(t \in L_{\nu}\left(x_{i}, v, d\right) \mid x_{i}\right)
$$

On the other hand, note that:

$$
\mathbb{P}_{\nu}\left(x_{-i} \in H_{\nu}\left(t, v, d_{-i}\right) \mid t\right)=\Pi_{j \neq i} \Phi_{j}^{v}\left(d_{j}\right)
$$

Consequently, we have:

$$
\mathbb{P}_{\nu}\left(x_{-i} \in H_{\nu}\left(x_{i}, v, d_{-i}\right) \mid x_{i}\right)=\int_{-\frac{1}{2}}^{\frac{1}{2}} \mathbb{P}_{\nu}\left(t \in L_{\nu}\left(x_{i}, v,-l\right) \mid x_{i}\right) \Pi_{j \neq i} \Phi_{j}^{v}\left(d_{j}+l\right) d l .
$$


This yields, applying Equation (12) and the fact that $\nu<\nu(\delta)$,

$$
\mathbb{P}_{\nu}\left(x_{-i} \in H_{\nu}\left(x_{i}, v, d_{-i}\right) \mid x_{i}\right) \geq \int_{-\frac{1}{2}}^{\frac{1}{2}} \phi_{i}^{v}(l) \Pi_{j \neq i} \Phi_{j}^{v}\left(d_{j}+l\right) d l-\delta .
$$

It now suffices to recall that by construction of $s_{c}^{\theta}(\bar{\nu}, \nu, v)$ (Lemma 1$)$ :

$$
\mathbb{P}_{\bar{\nu}}^{v}\left(s_{-i}^{\theta}(\bar{\nu}, v)\left(x_{-i}\right) \leq a_{-i} \mid x\left(a_{i}, s^{\theta}(\bar{\nu}, v)\right)\right)=\mathbb{P}_{\nu}^{v}\left(s_{c,-i}^{\theta}(\bar{\nu}, \nu, v)\left(x_{-i}\right) \leq a_{-i} \mid x\left(a_{i}, s_{c}^{\theta}(\bar{\nu}, \nu, v)\right)\right),
$$

and that, by Remark 1 ,

$\mathbb{P}_{\nu}^{v}\left(s_{c,-i}^{\theta}(\bar{\nu}, \nu, v)\left(x_{-i}\right) \leq a_{-i} \mid x\left(a_{i}, s_{c}^{\theta}(\bar{\nu}, \nu, v)\right)\right)=\int_{-\frac{1}{2}}^{\frac{1}{2}} \phi_{i}\left(\eta_{i}\right) \Pi_{j \neq i} \Phi_{j}^{v}\left(\kappa\left(a_{j}, \bar{\nu}, v\right)-\kappa\left(a_{i}, \bar{\nu}, v\right)+\eta_{i}\right) d \eta_{i}$, to conclude the proof.

Lemma 6. For any $\varepsilon>0$, there exists $\nu_{2}(\varepsilon)>0$ such that, for each $\nu<\nu_{2}(\varepsilon)$, the following property holds for each $\lambda \in[-1,1], i \in I, a_{i} \in A_{i}^{+} \backslash \max A_{i}$ and $x_{i} \in \mathbb{R}^{N}$ with $s_{i}(\lambda, \bar{\nu}, \nu)\left(x_{i}\right) \leq$ $a_{i}$. There exists a unit vector $\tilde{v} \in V$ such that for each $j \neq i, a_{j} \in A_{j}^{+}$and $x_{j} \in \mathbb{R}^{N}$ satisfying:

$$
x_{j} \in H_{\nu}\left(x_{i}, \tilde{v}, \kappa\left(a_{j}, \bar{\nu}, \tilde{v}\right)-\kappa\left(a_{i}, \bar{\nu}, \tilde{v}\right)-\varepsilon\right) \cap B^{\nu}\left(x_{i}\right),
$$

we have:

$$
s_{j}(\lambda, \bar{\nu}, \nu)\left(x_{j}\right) \leq a_{j}
$$

Proof of Lemma 6. We first establish Claim 1 below.

Claim 1. For any $\varepsilon>0$, there exists $\nu_{3}(\varepsilon)>0$ such that the following property holds for all $\nu<\nu_{3}(\varepsilon), x_{i} \in R^{N}, \tilde{t} \in[0,1]$ and $\kappa \in[0, C]$ with $\left\|\theta(\tilde{t})-x_{i}\right\| \in\left[\frac{\sqrt{\nu}}{2}, \sqrt{\nu}+\kappa \nu\right]$. For all $\kappa^{\prime} \in[0, C]$ and $x_{j} \in R^{N}$ such that:

$$
x_{j} \in H_{\nu}\left(x_{i}, \tilde{v}, \kappa^{\prime}-\kappa-\varepsilon\right) \cap B^{\nu}\left(x_{i}\right),
$$

with $\tilde{v}=\frac{x_{i}-\theta(\tilde{t})}{\left\|x_{i}-\theta(\tilde{t})\right\|}$, we have:

$$
\left\|\theta(\tilde{t})-x_{j}\right\| \leq \sqrt{\nu}+\left(\kappa^{\prime}-\frac{\varepsilon}{2}\right) \nu .
$$

Proof of Claim 1. We begin with the following remark.

Remark 3. Fix some $x_{i} \in \mathbb{R}^{N}$ and $\tilde{t} \in[0,1]$ with $\left\|\theta(\tilde{t})-x_{i}\right\| \geq \frac{\sqrt{\nu}}{2}$. Let $\tilde{v} \in \mathbb{R}^{N}$ be defined by $\tilde{v}=\frac{x_{i}-\theta(\tilde{t})}{\left\|x_{i}-\theta(\tilde{t})\right\|}$. For all $\nu>0$ and $d \in \mathbb{R}$, the following two properties are satisfied. 
1. If $d<-1, H_{\nu}\left(x_{i}, \tilde{v}, d\right) \cap B^{\nu}\left(x_{i}\right)=\emptyset$.

2. If $d \geq-1$, we have:

$$
\left\|\theta(\tilde{t})-x_{j}\right\|^{2} \leq\left(\left\|\theta(\tilde{t})-x_{i}\right\|+d \nu\right)^{2}+\nu^{2}
$$

for all $x_{j} \in H_{\nu}\left(x_{i}, \tilde{v}, d\right) \cap B^{\nu}\left(x_{i}\right)$.

Proof of Remark 3. Fix some $x_{i} \in \mathbb{R}^{N}, \tilde{t} \in[0,1], \nu>0$ and $d \in \mathbb{R}$. By Pythagorean theorem, for all $x_{j} \in L_{\nu}\left(x_{i}, \tilde{v}, d\right)$ (with $\left.\tilde{v}=\frac{x_{i}-\theta(\tilde{t})}{\left\|x_{i}-\theta(\tilde{t})\right\|}\right)$,

$$
(d \nu)^{2}+\left\|x_{j}-\left(x_{i}+(d \nu) \tilde{v}\right)\right\|^{2}=\left\|x_{j}-x_{i}\right\|^{2}
$$

For all $x_{j} \in B^{\nu}\left(x_{i}\right),\left\|x_{j}-x_{i}\right\|^{2} \leq \nu^{2}$. Hence, using Equation (13), we deduce that

$$
\left\|x_{j}-\left(x_{i}+(d \nu) \tilde{v}\right)\right\| \leq \nu
$$

for all $x_{j} \in L_{\nu}\left(x_{i}, \tilde{v}, d\right) \cap B^{\nu}\left(x_{i}\right)$ and that $L_{\nu}\left(x_{i}, \tilde{v}, d\right) \cap B^{\nu}\left(x_{i}\right)=\emptyset$ if $d \notin[-1,1]$, which establishes Point 1. Now assume that $d \geq-1$. By Pythagorean theorem, for each $x_{j} \in$ $L_{\nu}\left(x_{i}, \tilde{v}, d\right)$,

$$
\left\|\theta(\tilde{t})-x_{j}\right\|^{2}=\left\|\theta(\tilde{t})-\left(x_{i}+(d \nu) \tilde{v}\right)\right\|^{2}+\left\|\left(x_{i}+(d \nu) \tilde{v}\right)-x_{j}\right\|^{2} .
$$

Notice that by the definition of $\tilde{v}$,

$$
\left\|\theta(\tilde{t})-\left(x_{i}+(d \nu) \tilde{v}\right)\right\|=\left\|\theta(\tilde{t})-x_{i}\right\|+d \nu .
$$

Thus,

$$
\left\|\theta(\tilde{t})-x_{j}\right\|^{2}=\left(\left\|\theta(\tilde{t})-x_{i}\right\|+d \nu\right)^{2}+\left\|x_{j}-\left(x_{i}+(d \nu) \tilde{v}\right)\right\|^{2} .
$$

Consequently, by Equation (14),

$$
\left\|\theta(\tilde{t})-x_{j}\right\|^{2} \leq\left(\left\|\theta(\tilde{t})-x_{i}\right\|+d \nu\right)^{2}+\nu^{2}
$$

which concludes the proof.

We now conclude the proof of Claim 1. Fix $\varepsilon>0, \kappa, \kappa^{\prime} \in[0, C], x_{i} \in \mathbb{R}^{N}, \tilde{t} \in[0,1]$ with $\left\|\theta(\tilde{t})-x_{i}\right\| \in\left[\frac{\sqrt{\nu}}{2}, \sqrt{\nu}+\kappa \nu\right]$ and let $\tilde{v}=\frac{x_{i}-\theta(\tilde{t})}{\left\|x_{i}-\theta(\tilde{t})\right\|}$. We distinguish two cases. First assume that $\kappa^{\prime}-\kappa-\varepsilon<-1$. By Remark 3, we know that:

$$
H_{\nu}\left(x_{i}, \tilde{v}, \kappa^{\prime}-\kappa-\varepsilon\right) \cap B^{\nu}\left(x_{i}\right)=\emptyset .
$$


Now assume that $\kappa^{\prime}-\kappa-\varepsilon \geq-1$. In that case, by Remark 3,

$$
\left\|\theta(\tilde{t})-x_{j}\right\|^{2} \leq\left(\left\|\theta(\tilde{t})-x_{i}\right\|+\left(\kappa^{\prime}-\kappa-\varepsilon\right) \nu\right)^{2}+\nu^{2},
$$

for each $x_{j} \in H_{\nu}\left(x_{i}, \tilde{v}, \kappa^{\prime}-\kappa-\varepsilon\right) \cap B^{\nu}\left(x_{i}\right)$. Since $\left\|\theta(\tilde{t})-x_{i}\right\| \geq \frac{\sqrt{\nu}}{2}$, we have: $\left\|\theta(\tilde{t})-x_{i}\right\|+$ $\left(\kappa^{\prime}-\kappa-\varepsilon\right) \nu>0$ when $\nu$ is sufficiently small (i.e., such that $\frac{\sqrt{\nu}}{2}>\nu$ ), which means that $\left(\left\|\theta(\tilde{t})-x_{i}\right\|+\left(\kappa^{\prime}-\kappa-\varepsilon\right) \nu\right)^{2}$ increases with $\left\|\theta(\tilde{t})-x_{i}\right\|$. This yields, using the fact that $\left\|\theta(\tilde{t})-x_{i}\right\| \leq \sqrt{\nu}+\kappa \nu$

$$
\left\|\theta(\tilde{t})-x_{j}\right\|^{2} \leq\left(\sqrt{\nu}+\left(\kappa^{\prime}-\varepsilon\right) \nu\right)^{2}+\nu^{2}
$$

In addition, we have:

$$
\left(\sqrt{\nu}+\left(\kappa^{\prime}-\varepsilon\right) \nu\right)^{2}+\nu^{2}=\nu+2\left(\kappa^{\prime}-\varepsilon\right) \nu \sqrt{\nu}+\left(\left(\kappa^{\prime}-\varepsilon\right)^{2}+1\right) \nu^{2} .
$$

Consequently,

$\left(\sqrt{\nu}+\left(\kappa^{\prime}-\varepsilon\right) \nu\right)^{2}+\nu^{2}=\nu+2\left(\kappa^{\prime}-\frac{\varepsilon}{2}\right) \nu \sqrt{\nu}+\left(\kappa^{\prime}-\frac{\varepsilon}{2}\right)^{2} \nu^{2}+\left(\left(\kappa^{\prime}-\varepsilon\right)^{2}+1-\left(\kappa^{\prime}-\frac{\varepsilon}{2}\right)^{2}\right) \nu^{2}-\varepsilon \nu \sqrt{\nu}$ that is,

$$
\left(\sqrt{\nu}+\left(\kappa^{\prime}-\varepsilon\right) \nu\right)^{2}+\nu^{2}=\left(\sqrt{\nu}+\left(\kappa^{\prime}-\frac{\varepsilon}{2}\right) \nu\right)^{2}+\left(\left(\kappa^{\prime}-\varepsilon\right)^{2}+1-\left(\kappa^{\prime}-\frac{\varepsilon}{2}\right)^{2}\right) \nu^{2}-\varepsilon \nu \sqrt{\nu} .
$$

Note that:

$$
\left(\left(\kappa^{\prime}-\varepsilon\right)^{2}+1-\left(\kappa^{\prime}-\frac{\varepsilon}{2}\right)^{2}\right) \nu^{2}-\varepsilon \nu \sqrt{\nu}=\left(\frac{3 \varepsilon^{2}}{4}-\varepsilon \kappa^{\prime}+1\right) \nu^{2}-\varepsilon \nu \sqrt{\nu} \leq\left(\frac{3 \varepsilon^{2}}{4}+1\right) \nu^{2}-\varepsilon \nu \sqrt{\nu}
$$

We deduce that when $\nu$ is sufficiently small (compared to $\varepsilon$ ):

$$
\left(\left(\kappa^{\prime}-\varepsilon\right)^{2}+1-\left(\kappa^{\prime}-\frac{\varepsilon}{2}\right)^{2}\right) \nu^{2}-\varepsilon \nu \sqrt{\nu} \leq 0 .
$$

This finally yields, using Equation (15),

$$
\left\|\theta(\tilde{t})-x_{j}\right\|^{2} \leq\left(\sqrt{\nu}+\left(\kappa^{\prime}-\frac{\varepsilon}{2}\right) \nu\right)^{2},
$$

which concludes the proof of Claim 1.

Claim 2. For any $\varepsilon>0$, there exists $\nu_{4}(\varepsilon)>0$ such that for any $x_{i} \in \mathbb{R}^{N}, \tilde{t} \in[0,1]$ with $\left\|\theta(\tilde{t})-x_{i}\right\| \geq \frac{\sqrt{\nu}}{2}, x_{j} \in B^{\nu}\left(x_{i}\right)$ and $a_{j} \in \cup_{j \in I} A_{j}^{+}$,

$$
\left|\kappa\left(a_{j}, \tilde{v}\right)-\kappa\left(a_{j}, \tilde{v}^{\prime}\right)\right|<\frac{\varepsilon}{2},
$$

with $\tilde{v^{\prime}}=\frac{x_{j}-\theta(\tilde{t})}{\| x_{j}-\theta(\tilde{t} \|}$ and $\tilde{v}=\frac{x_{i}-\theta(\tilde{t})}{\| x_{i}-\theta(\tilde{t} \|}$. 
Proof of Claim 2. For each $a_{i} \in A_{i}^{+}$, the function $\tilde{v} \mapsto \kappa\left(a_{i}, \tilde{v}\right)$ is continuous and the set $V$ of unit vectors $\tilde{v} \in \mathbb{R}^{N}$ is compact. Hence, the function $\tilde{v} \mapsto \kappa\left(a_{i}, \tilde{v}\right)$ is uniformly continuous on $V$ and it suffices to establish Remark 4 below.

Remark 4. For any $\varepsilon>0$ there exists $\nu(\varepsilon)>0$ such that for any $x_{i} \in \mathbb{R}^{N}, \tilde{t} \in[0,1]$ with $\left\|x_{i}-\theta(\tilde{t})\right\| \geq \frac{\sqrt{\nu}}{2}$ and $x_{j} \in B^{\nu}\left(x_{i}\right)$

$$
\left\|\frac{x_{i}-\theta(\tilde{t})}{\left\|x_{i}-\theta(\tilde{t})\right\|}-\frac{x_{j}-\theta(\tilde{t})}{\left\|x_{j}-\theta(\tilde{t})\right\|}\right\|<\varepsilon .
$$

Proof of Remark 4. We have:

$$
\left\|\frac{x_{i}-\theta(\tilde{t})}{\left\|x_{i}-\theta(\tilde{t})\right\|}-\frac{x_{j}-\theta(\tilde{t})}{\left\|x_{j}-\theta(\tilde{t})\right\|}\right\|=\left\|\frac{x_{i}-x_{j}}{\left\|x_{i}-\theta(\tilde{t})\right\|}+\frac{\left(x_{j}-\theta(\tilde{t})\right)\left(\left\|x_{j}-\theta(\tilde{t})\right\|-\left\|x_{i}-\theta(\tilde{t})\right\|\right)}{\left\|x_{i}-\theta(\tilde{t})\right\|\left\|x_{j}-\theta(\tilde{t})\right\|}\right\| .
$$

Since $\left\|x_{i}-\theta(\tilde{t})\right\| \geq \frac{\sqrt{\nu}}{2}$

$$
\frac{\left\|x_{i}-x_{j}\right\|}{\left\|x_{i}-\theta(\tilde{t})\right\|} \leq 2 \sqrt{\nu}
$$

for all $x_{j} \in B^{\nu}\left(x_{i}\right)$. In addition, by the triangle inequality,

$$
\left\|x_{i}-\theta(\tilde{t})\right\|+\nu \geq\left\|x_{j}-\theta(\tilde{t})\right\| \geq\left\|x_{i}-\theta(\tilde{t})\right\|-\nu
$$

for all $x_{j} \in B^{\nu}\left(x_{i}\right)$. We deduce:

$$
\left\|\frac{\left(x_{j}-\theta(\tilde{t})\right)\left(\left\|x_{j}-\theta(\tilde{t})\right\|-\left\|x_{i}-\theta(\tilde{t})\right\|\right)}{\left\|x_{i}-\theta(\tilde{t}) \mid\right\| x_{j}-\theta(\tilde{t}) \|}\right\| \leq\left\|\frac{\nu\left(x_{j}-\theta(\tilde{t})\right)}{\left\|x_{i}-\theta(\tilde{t})\left|\|\mid\| x_{j}-\theta(\tilde{t}) \|\right.\right.}\right\| \leq 2 \sqrt{\nu} .
$$

Hence, Equation (16) yields:

$$
\left\|\frac{x_{i}-\theta(\tilde{t})}{\left\|x_{i}-\theta(\tilde{t})\right\|}-\frac{x_{j}-\theta(\tilde{t})}{\left\|x_{j}-\theta(\tilde{t})\right\|}\right\| \leq 4 \sqrt{\nu}
$$

which concludes the proof.

Let us now conclude the proof of Lemma 6. Notice that there exists $\nu^{\star}$ such that for all $\nu<\nu^{\star}, \frac{\sqrt{\nu}}{2}+\nu<\sqrt{\nu}$ and pick some $\nu<\min \left(\nu^{\star}, \nu_{3}(\varepsilon), \nu_{4}(\varepsilon)\right), i, \lambda \in[-1,1], a_{i} \in A_{i}^{+} \backslash \max A_{i}$ and $x_{i} \in \mathbb{R}^{N}$ with $s_{i}(\lambda, \bar{\nu}, \nu)\left(x_{i}\right) \leq a_{i}$. By construction, there exists some $\tilde{t} \in\left[0, \tilde{t}^{\star}+\lambda\right]$ such that $\left\|x_{i}-\theta(\tilde{t})\right\| \leq \sqrt{\nu}+\kappa\left(a_{i}, \bar{\nu}, \tilde{v}\right) \nu$ with $\tilde{v}=\frac{x_{i}-\theta(\tilde{t})}{\left\|x_{i}-\theta(\tilde{t})\right\|}$. We need to separate two cases. First assume that $\left\|\theta(\tilde{t})-x_{i}\right\| \leq \frac{\sqrt{\nu}}{2}$. Since $\nu<\nu^{\star}$, we have: $\left\|\theta(\tilde{t})-x_{j}\right\|<\sqrt{\nu}$, for all $x_{j} \in B^{\nu}\left(x_{i}\right)$, which by construction ensures that $s_{j}(\lambda, \bar{\nu}, \nu)\left(x_{j}\right) \leq a_{j}$, for all $a_{j} \in A_{j}^{+}$. Now, assume that $\left\|\theta(\tilde{t})-x_{i}\right\| \geq \frac{\sqrt{\nu}}{2}$. Let $a_{j} \in A_{j}^{+}$and $x_{j} \in \mathbb{R}^{N}$ be such that $x^{j} \in H_{\nu}\left(x_{i}, \tilde{v}, \kappa\left(a_{j}, \bar{\nu}, \tilde{v}\right)-\right.$ $\left.\kappa\left(a_{i}, \bar{\nu}, \tilde{v}\right)-\varepsilon\right) \cap B^{\nu}\left(x_{i}\right)$. By Claim 1, we have:

$$
\left\|x_{j}-\theta(\tilde{t})\right\| \leq \sqrt{\nu}+\left(\kappa\left(a_{j}, \bar{\nu}, \tilde{v}\right)-\frac{\varepsilon}{2}\right) \nu .
$$


By Claim 2, this implies:

$$
\left\|x_{j}-\theta(\tilde{t})\right\| \leq \sqrt{\nu}+\kappa\left(a_{j}, \bar{\nu}, \tilde{v}^{\prime}\right) \nu
$$

with $\tilde{v}^{\prime}=\frac{x_{j}-\theta(\tilde{t})}{\left\|x_{j}-\theta(\tilde{t})\right\|}$, which concludes the proof.

We now conclude the proof of Proposition 2. Notice that the function $\left(x_{i}, \tilde{v}, d_{-i}\right) \mapsto$ $\mathbb{P}_{\nu}\left(x_{-i} \in H_{\nu}\left(x_{i}, \tilde{v}, d_{-i}\right) \mid x_{i}\right)$ is continuous on the compact set $B^{\bar{\mu}}(\Theta) \times V \times[-1,1]^{|I-1|}$. Hence, it is uniformly continuous on $B^{\bar{\mu}}(\Theta) \times V \times[-1,1]^{|I-1|}$ and there exists a function $\varepsilon: \mathbb{R}_{+}^{\star} \rightarrow \mathbb{R}_{+}^{\star}$ such that:

$$
\mathbb{P}_{\nu}\left(x_{-i} \in H_{\nu}\left(x_{i}, \tilde{v}, d_{-i}-\varepsilon(\delta)\right) \mid x_{i}\right) \geq \mathbb{P}_{\nu}\left(x_{-i} \in H_{\nu}\left(x_{i}, \tilde{v}, d_{-i}\right) \mid x_{i}\right)-\delta .
$$

for all $\delta>0, d_{-i} \in[-1,1]^{|I-1|}, v \in V$ and $x_{i} \in B^{\bar{\mu}}(\Theta)$. Now, fix some $\delta>0, \lambda \in[-1,1], \nu<$ $\min \left(\nu_{1}\left(\frac{\delta}{2}\right), \nu_{2}\left(\varepsilon\left(\frac{\delta}{2}\right)\right), a_{i} \in A_{i}^{+} \backslash \max A_{i}\right.$ and $x_{i} \in \mathbb{R}^{N}$ such that $s_{i}(\lambda, \bar{\nu}, \nu)\left(x_{i}\right) \leq a_{i}$. By Lemma 6 , there exists a unit vector $\tilde{v} \in V$ such that:

$$
\mathbb{P}_{\nu}\left(s_{-i}(\lambda, \bar{\nu}, \nu)\left(x_{-i}\right) \leq a_{-i} \mid x_{-i}\right) \geq \mathbb{P}_{\nu}\left(x_{-i} \in H_{\nu}\left(x_{i}, \tilde{v}, \kappa\left(a_{-i}, \bar{\nu}, \tilde{v}\right)-\kappa\left(a_{i}, \bar{\nu}, \tilde{v}\right)\right) \mid x_{i}\right)-\frac{\delta}{2} .
$$

In addition, by Lemma 5,

$$
\mathbb{P}_{\nu}\left(x_{-i} \in H_{\nu}\left(x_{i}, \tilde{v}, \kappa\left(a_{-i}, \bar{\nu}, \tilde{v}\right)-\kappa\left(a_{i}, \bar{\nu}, \tilde{v}\right)\right) \mid x_{i}\right) \geq \mathbb{P}_{\nu}^{\tilde{v}}\left(s^{\theta}(\bar{\nu}, \tilde{v})\left(x_{-i}\right) \leq a_{-i} \mid x\left(a_{i}, s^{\theta}(\bar{\nu}, \tilde{v})\right)\right)-\frac{\delta}{2}
$$

Combining the two equations above finally yields the desired result.

\subsection{Proof of Proposition 3.}

Recall that, by Proposition $1, x\left(a_{i}, s^{\theta}(\bar{\nu}, v)\right)>\tilde{t}^{\star}+\lambda^{\star}$, for each $i \in I, a_{i} \in A_{i}^{+}$and unit vector $v \in V$. Hence, for all $\lambda \leq 0$ and $\tilde{t} \in\left[0, \tilde{t}^{\star}+\lambda\right], x\left(a_{i}, s^{\theta}(\bar{\nu}, v)\right)-\tilde{t}>\lambda^{\star}$. Consequently, by strict monotonicity,

$$
\Delta u_{i}^{\theta}\left(a^{\prime} \rightarrow a_{i}, a_{-i}, \tilde{t}\right) \geq \Delta u_{i}^{\theta}\left(a_{i}^{\prime} \rightarrow a_{i}, a_{-i}, x\left(a_{i}, s^{\theta}(\bar{\nu}, v)\right)\right)+\lambda^{\star} K
$$

for each $a_{i}^{\prime} \in A_{i}$ with $a_{i}^{\prime}>a_{i}$ and each unit vector $v \in V$. Since the set $B^{\bar{\mu}}(\theta)$ is compact and the function $u_{i}(a, \cdot)$ is continuous, there exists a function $\xi: \mathbb{R}_{+}^{\star} \rightarrow \mathbb{R}_{+}^{\star}$ such that for any given $\varepsilon>0, a \in A$ and $t, t^{\prime} \in B^{\bar{\mu}}(\Theta)$ with ||$t-t^{\prime}||<\xi(\varepsilon)$, we have: $\left|u_{i}(a, t)-u_{i}\left(a, t^{\prime}\right)\right|<\varepsilon$. Notice that there exists $\nu^{\star}>0$ such that for each $\nu<\nu^{\star}, \sqrt{\nu}+C \nu<\xi\left(\frac{\lambda^{\star} K}{4}\right)-\frac{\nu}{2}$ (where we recall that $\left.C=\sum_{i \in I}\left|A_{i}\right|\right)$. Now, fix some $\nu<\nu^{\star}, \lambda \leq 0, a_{i} \in A_{i}^{+}$and $x_{i} \in \mathbb{R}^{N}$ with 
$s(\lambda, \bar{\nu}, \nu)\left(x_{i}\right) \leq a_{i}$. By construction of the strategy profile $s(\lambda, \bar{\nu}, \nu)$, we know that there exists some $\tilde{t} \in\left[0, t^{\star}+\lambda\right]$ such that:

$$
\left\|x_{i}-\theta(\tilde{t})\right\|<\sqrt{\nu}+C \nu
$$

Since $\nu<\nu^{\star}$, this implies:

$$
\left\|x_{i}-\theta(\tilde{t})\right\| \leq \xi\left(\frac{\lambda^{\star} K}{4}\right)-\frac{\nu}{2} .
$$

Hence, by the triangle inequality, for all $t \in B^{\frac{\nu}{2}}\left(x_{i}\right),\|t-\theta(\tilde{t})\|<\xi\left(\frac{\lambda^{\star} K}{4}\right)$. Consequently, for all $a \in A$ and $t \in B^{\frac{\nu}{2}}\left(x_{i}\right)$,

$$
\left\|u_{i}(a, t)-u_{i}(a, \theta(\tilde{t}))\right\|<\frac{\lambda^{\star} K}{4} .
$$

Thus for all $a_{-i} \in A_{-i}$ and $t \in B^{\frac{\nu}{2}}\left(x_{i}\right)$,

$$
\Delta u_{i}\left(a_{i}^{\prime} \rightarrow a_{i}, a_{-i}, t\right) \geq \Delta u_{i}^{\theta}\left(a^{\prime} \rightarrow a_{i}, a_{-i}, \tilde{t}\right)-\frac{\lambda^{\star} K}{2},
$$

for all actions $a_{i}^{\prime} \in A_{i}^{+}$with $a_{i}^{\prime}>a_{i}$. Putting Equations (17) and (18) together, we deduce that for all $\lambda \leq 0, \nu<\nu^{\star}$ and $x_{i} \in \mathbb{R}^{N}$ with $s(\lambda, \bar{\nu}, \nu)\left(x_{i}\right) \leq a_{i}$ :

$$
\Delta u_{i}\left(a_{i}^{\prime} \rightarrow a_{i}, a_{-i}, t\right) \geq \Delta u_{i}^{\theta}\left(a_{i}^{\prime} \rightarrow a_{i}, a_{-i}, x\left(a_{i}, s^{\theta}(\bar{\nu}, v)\right)\right)+\frac{\lambda^{\star} K}{2},
$$

for all $t \in B^{\frac{\nu}{2}}\left(x_{i}\right), a_{i}^{\prime} \in A_{i}^{+}$with $a_{i}^{\prime}>a_{i}$ and unit vectors $v \in V$, which concludes the proof. 